\title{
A mean field limit for the Vlasov-Poisson system
}

\author{
Dustin Lazarovici* and Peter Pickl ${ }^{\dagger}$ \\ Mathematisches Institut, Ludwig-Maximilians Universität \\ Theresienstr. 39, 80333 Munich, Germany.
}

June 2, 2016

\begin{abstract}
We present a probabilistic proof of the mean field limit and propagation of chaos $N$-particle systems in three dimensions with positive (Coulomb) or negative (Newton) $1 / r$ potentials scaling like $1 / N$ and an $N$-dependent cut-off which scales like $N^{-1 / 3+\epsilon}$. In particular, for typical initial data, we show convergence of the empirical distributions to solutions of the Vlasov-Poisson system with either repulsive electrical or attractive gravitational interactions.
\end{abstract}

\section{Introduction}

We are interested in a microscopic derivation of the nonrelativistic Vlasov-Poisson system. This equation describes a plasma of identical charged particles with electrostatic or gravitational interactions

$$
\partial_{t} f+p \cdot \nabla_{q} f+\left(k * \rho_{t}\right) \cdot \nabla_{p} f=0,
$$

where $k$ is the (Coulomb) kernel

$$
k(q):=\sigma \frac{q}{|q|^{3}}, \quad \sigma=\{ \pm 1\},
$$

and

$$
\rho_{t}(q)=\rho\left[f_{t}\right](q)=\int f(t, q, p) \mathrm{d}^{3} p
$$

is the charge density induced by the distribution $f(t, p, q) \geq 0$.

Units are chosen such that all constants, in particular the mass and charge of the particles, are equal to 1 . The case $\sigma=+1$ corresponds to electrostatic (repulsive) interactions, while $\sigma=-1$ describes gravitational (attractive) interactions. In the gravitational case, (1-3) is also known as the Vlasov-Newton equation. For simplicity, we focus on the 3 -dimensional case, generalization to arbitrary dimensions $d \geq 2$ is straightforward.

\footnotetext{
*dustin.lazarovici@live.com

${ }^{\dagger}$ pickl@math.lmu.de
} 


\subsection{Previous results}

While the existence theory of the Vlasov-Poisson dynamics is well understood - we will cite the pertinent results below - its microscopic derivation is still an open problem. To our knowledge, the first paper to discuss a mathematically rigorous derivation of Vlasov equations is Neunzert and Wick, 1974 [15. Better known are the publications of Braun and Hepp, 1977 [2] and Dobrushin, 1979 [3], as well as the later exposition of Neunzert, 1984 [14]. For a general overview of the topic, we refer the reader to the book of Spohn [18].

Rather than the Vlasov-Poisson equation, the papers of Neunzert, Braun and Hepp and Dobrushin treat simplified models with Lipschitz continuous forces $k \in W^{1, \infty}=\left\{k \in C^{1}\left(\mathbb{R}^{d}\right):\|k\|_{\infty}+\|\nabla k\|_{\infty}<\infty\right\}$. The last few years have seen great progress in treating mean field limits for singular forces up to but not including the Coulomb case. In particular, Hauray and Jabin, 2013, discuss force kernels bounded as $|k(q)| \leq \frac{C}{|q|^{\alpha}}$ with $\alpha<d-1$ in $d \geq 3$ dimensions [6]. For $1<\alpha<d-1$, they perform the mean field limit for typical initial data and an $N$ dependent cut-off that can be chosen as small as $N^{-\frac{1}{2 d}}$ for $\alpha \nearrow d-1$. For $\alpha<1$, they are even able to prove molecular chaos with no cutoff at all. Unfortunately, their method fails precisely at the Coulomb threshold $\alpha=d-1$.

In contrast, Kiessling, 2014 proves a non-quantitative approximation result including the Coulomb singularity under the assumption of an (uniform in $N$ ) a priori bound on the microscopic forces. The status of this assumption, however, whether it is satisfied for generic initial data or not, remains open [10.

Recently, Boers and Pickl proposed a novel method for deriving mean field equations which is designed for stochastic initial conditions, thus aiming directly at a typicality result. With this method, they were able to improve the cut-off near the Coulomb case to $\sim N^{-\frac{1}{d}}[1$.

The aim of this paper is to extend the method of Boers and Pickl to include the Coulomb singularity in the large $N$ limit, thus aiming at a microscopic derivation of the Vlasov-Poisson dynamics. The Coulomb case is qualitatively different from the previously treated interactions since the mean field force $k * \rho$ is no longer Lipschitz, in general, even for bounded $\rho$. However, we will show how this critical case can be treated by exploiting the second order nature of the dynamics and introducing an anisotropic scaling of the relevant metric. Moreover, we optimize the method in such a way as to achieve a rate of convergence that can be faster than any inverse power of $N$, depending on decay properties of the initial distribution $f_{0}$.

An alternative proof, based on similar modifications of the Wasserstein distance, is simultaneously proposed in 11. The result presented here, however, allows for a significantly smaller cutoff $N^{-\frac{1}{d}+\epsilon}$ to be compared with $N^{-\frac{1}{d(d+2)}}+\epsilon$. 


\section{The microscopic model}

Since the Coulomb kernel is strongly singular at the origin, we will require a regularization on the microscopic level. For $N \in \mathbb{N}$ and $\delta \geq 0$, we consider

$$
k_{\delta}^{N}(q):=\sigma\left\{\begin{aligned}
\frac{q}{|q|^{3}} & , \text { if }|q| \geq N^{-\delta} \\
q N^{3 \delta} & , \text { else. }
\end{aligned}\right.
$$

On $\mathbb{R}^{3} \backslash\{0\}$ this converges to the Coulomb kernel (2) as $N \rightarrow \infty$. Of course, the $N$-dependence of the force thus introduced is a technical necessity rather than a realistic physical model, though similar regularizations are commonly used in numerical computations.

In the mean field scaling, the equations of motion for the regularized $N$-particle system are given by

$$
\left\{\begin{array}{l}
\dot{q}_{i}(t)=p_{i}(t) \\
\dot{p}_{i}(t)=\frac{1}{N} \sum_{j=1}^{N} k_{\delta}^{N}\left(q_{i}-q_{j}\right),
\end{array}\right.
$$

for $i \in 1, \ldots, N$. Since the vector field is Lipschitz for fixed $\delta, N$, we have global existence and uniqueness of solutions and hence an $N$-particle Hamiltonian flow which we denote by ${ }^{N} \Psi_{t, s}(Z)=\left({ }^{N} \Psi_{t, s}^{1}(Z),{ }^{N} \Psi_{t, s}^{2}(Z)\right) \in$ $\mathbb{R}^{3 N} \times \mathbb{R}^{3 N}$. Introducing the $N$-particle force $K_{\delta}^{N}: \mathbb{R}^{3 N} \rightarrow \mathbb{R}^{3 N}$ given by

$$
\left(K_{\delta}^{N}\left(q_{1}, . ., q_{N}\right)\right)_{i}:=\frac{1}{N} \sum_{j=1}^{N} k_{\delta}^{N}\left(q_{i}-q_{j}\right), \quad i=1, . ., N,
$$

we can also characterize ${ }^{N} \Psi_{t, s}$ as the solution of

$$
\frac{\mathrm{d}}{\mathrm{d} t}\left(\Psi_{t, s}^{1}(Z), \Psi_{t, s}^{2}(Z)\right)=\left(\Psi_{t, s}^{2}(Z), K_{\delta}^{N}\left(\Psi_{t, s}^{1}(Z)\right)\right), \Psi_{s, s}(Z)=Z .
$$

Finally, if ${ }^{N} \Psi_{t, 0}(Z)=\left(q_{i}(t), p_{i}(t)\right)_{i=1, \ldots, N}$, we define the corresponding microscopic or empirical density by

$$
\mu_{t}^{N}[Z]=\mu_{0}^{N}\left[\Psi_{t, 0}(Z)\right]:=\frac{1}{N} \sum_{i=1}^{N} \delta\left(\cdot-q_{i}(t)\right) \delta\left(\cdot-p_{i}(t)\right) .
$$

Our aim is to show that for typical $Z$, the empirical density $\mu_{t}^{N}[Z]$ converges to a solution $f_{t}$ of the Vlasov-Poisson equation as $N \rightarrow \infty$.

Of course, more general cut-offs can be considered. In the literature, the following nomenclature has been established (see e.g. [6]):

Definition 2.1. A pair-interaction defined by a kernel $k: \mathbb{R}^{d} \rightarrow \mathbb{R}^{d}$ satisfies a $S^{\alpha}$-condition, if

$$
\exists c>0, \forall q \in \mathbb{R}^{d} \backslash\{0\} \quad|k(q)| \leq \frac{c}{|q|^{\alpha}}, \quad|\nabla k| \leq \frac{c}{|q|^{\alpha+1}} .
$$


Introducing a cut-off of order $N^{-\delta}$ near the origin, the regularized force kernel $k_{\delta}^{N}$ satisfies a $\left(S_{\delta}^{\alpha}\right)$-condition if

i) $\quad k$ satisfies a $\left(S^{\alpha}\right)$ condition,

ii) $k_{\delta}^{N}(q)=k(q)$ for $|q| \geq N^{-\delta}$,

iii) $\left|k_{\delta}^{N}(q)\right| \leq N^{\delta \alpha}$ for all $|q|<N^{-\delta}$.

In addition, we shall require that

$$
\text { iv) }\left|\nabla k_{\delta}^{N}(q)\right| \leq N^{\delta(\alpha+1)} \text { for all }|q|<N^{-\delta},
$$

which assures that the regularization around the origin is not too erratic.

Within this setting, we thus consider 3-dimensional force kernels satisfying a $\left(S_{\delta}^{\alpha}\right)$ condition with $\alpha=2$ and the additional assumption $i v$ ). The lower bound on the cut-off will later be determined as $\delta<\frac{1}{3}$. Moreover, we shall adopt the convention $k_{\delta}^{N}(0)=0$, meaning that the microscopic dynamics do not contain self-interactions. The reader is free to think of (4) as defining the microscopic model or consider another regularization of his liking that satisfies the above assumptions.

\subsection{The regularized Vlasov-Poisson equation}

For any $\delta>0$ and $N \in \mathbb{N} \cup\{\infty\}$, we also consider the corresponding mean field equation

$$
\partial_{t} f+p \cdot \nabla_{q} f+\left(k_{\delta}^{N} * \rho_{t}\right) \cdot \nabla_{p} f=0 .
$$

For (formally) $N=\infty$, this reduces to the Vlasov-Poisson equation (11). For a fixed initial distribution $f_{0} \in L^{\infty}\left(\mathbb{R}^{3} \times \mathbb{R}^{3}\right)$ with $f_{0} \geq 0$ and $\int f=1$ we denote by $f_{t}^{N}$ the unique solution of (10) with initial datum $f_{t}^{N}(0, \cdot, \cdot)=f_{0}$.

\subsection{Method of characteristics}

It is convenient to consider the characteristic flow of the mean field system. For $N \in \mathbb{N}, \delta>0$ and $\rho \in L^{1}\left(\mathbb{R}^{3}\right)$, we define $\widehat{K}_{\delta}^{N}(\cdot ; \rho)$ : $\mathbb{R}^{3} \times \mathbb{R}^{3} \rightarrow \mathbb{R}^{3} \times \mathbb{R}^{3}$ by

$$
\widehat{K}_{\delta}^{N}(q, p ; \rho):=\left(p, k_{\delta}^{N} * \rho(q)\right) .
$$

Then, the (regularized) Vlasov-Poisson equation (10) with initial $f_{0}$ is equivalent to the following system of integro-differential equations:

$$
\left\{\begin{array}{l}
\frac{\mathrm{d}}{\mathrm{d} t} \varphi_{t, s}^{N}\left(z ; f_{0}\right)=\widehat{K}_{\delta}^{N}\left(\varphi_{t, s}^{N}\left(z ; f_{0}\right) ; \rho_{t}^{N}\right) \\
\rho_{t}^{N}(q)=\int f^{N}(t, q, p) \mathrm{d}^{3} p \\
f^{N}(t, \cdot)=\varphi_{t, s}^{N}\left(\cdot ; f_{0}\right) \# f_{s}^{N} \\
\varphi_{s, s}^{N}\left(z ; f_{0}\right)=z
\end{array}\right.
$$


Here, $\varphi(\cdot) \# f$ denotes the image-measure of $f$ under $\varphi$, defined by $\varphi \# f(A)=f\left(\varphi^{-1}(A)\right)$ for any Borel set $A \subseteq \mathbb{R}^{6}$.

In other words, we have non-linear time-evolution in which $\varphi_{t, s}^{N}\left(\cdot ; f_{0}\right)$ is the one-particle flow induced by the mean field dynamics with initial distribution $f_{0}$, while, in turn, $f_{0}$ is transported with the flow $\varphi_{t, s}^{N}$. Due to the semi-group property $\varphi_{t, s^{\prime}}^{N} \circ \varphi_{s^{\prime}, s}^{N}=\varphi_{t, s}^{N}$ it generally suffices to consider the initial time $s=0$.

The method of characteristics can also be though of as establishing a kind of duality between the (rescaled) Newtonian dynamics (5) and the Vlasov equation (10). Indeed, observing that the microscopic force can be written as

$$
\frac{1}{N} \sum_{j=1}^{N} k_{\delta}^{N}\left(q_{i}-q_{j}\right)=k_{\delta}^{N} * \mu_{t}^{N}[Z]\left(q_{i}\right),
$$

one easily checks that $\Psi_{t, 0}(Z)$ solves (5) with $\Psi_{0}(Z)=0$ if and only if $g_{t}=\mu_{0}^{N}\left[\Psi_{t, 0}(Z)\right]$ is a weak solution of (10) with $g_{0}=\mu_{0}^{N}[Z]$.

This relation is often used to translate the microscopic dynamics into a Vlasov equation, allowing to treat $\mu_{t}^{N}[Z]$ and $f_{t}$ on the same footing. Here, we will go the opposite way, so to speak, and transform the mean field dynamics into corresponding $N$ particle dynamics. To this end, we consider the lift of $\varphi_{t, s}^{N}(\cdot)$ to the $N$-particle phase-space, which we denote by ${ }^{N} \Phi_{t, s}$. That is, for $f_{0} \in L^{1}\left(\mathbb{R}^{6}\right)$ and $Z=\left(q_{i}, p_{i}\right)_{1 \leq i \leq N}$, we define

$$
{ }^{N} \Phi_{t, s}\left(Z ; f_{0}\right):=\left(\varphi_{t, s}^{N}\left(q_{1}, p_{1} ; f_{0}\right), \ldots, \varphi_{t, s}^{N}\left(q_{N}, p_{N} ; f_{0}\right)\right) .
$$

Denoting by $\bar{K}: \mathbb{R}^{3 N} \rightarrow \mathbb{R}^{3 N}$ the lift of the mean field force to the $N$-particle phase-space, i.e.

$$
\left(\bar{K}_{t}(Z)\right)_{i}:=k_{\delta}^{N} * \rho\left[f_{t}^{N}\right]\left(z_{i}\right), \quad Z=\left(z_{1}, \ldots, z_{N}\right),
$$

the flow ${ }^{N} \Phi_{t, s}(Z)=\left({ }^{N} \Phi_{t, s}^{1}(X),{ }^{N} \Phi_{t, s}^{2}(X)\right)$ can also be characterized as the solution of the non-autonomous differential equation

$$
\frac{\mathrm{d}}{\mathrm{d} t}\left(\Phi_{t, s}^{1}(Z), \Phi_{t, s}^{2}(Z)\right)=\left(\Phi_{t, s}^{2}(Z), \bar{K}_{t}\left(\Phi_{t, s}^{1}(Z)\right)\right), \Phi_{s, s}(Z)=Z
$$

to be compared with (7). Finally, we introduce the corresponding empirical density

$$
\mu_{0}^{N}\left[\Phi_{t, 0}(Z)\right]=\varphi_{t, 0}^{N} \# \mu_{0}^{N}[Z],
$$

pertaining to the mean field dynamics with (random) initial conditions $Z \in \mathbb{R}^{6 N}$.

In summary, for fixed $f_{0}$ and $N \in \mathbb{N}$, we consider for any initial configuration $Z \in \mathbb{R}^{6 N}$ two different time-evolutions: ${ }^{N} \Psi_{t, 0}(Z)$, given by the microscopic equations (5) and ${ }^{N} \Phi_{t, 0}(Z)$, given by the time-dependent mean field force generated by $f_{t}^{N}$. We are going to show that for typical $Z$, the two time-evolutions or close in an appropriate sense. 


\section{Existence of solutions}

For the well-posedness of the Vlasov-Poisson system, we can rely on various results establishing global existence and uniqueness of (weak and strong) solutions under fairly mild conditions on the initial configuration $f_{0}$ (Pfaffelmoser, 1990 [16, Schaeffer, 1991 [17], Lions and Perthame, 1991 [12], Horst, 1993 [8]). For our purposes, the following existence result due to Lions and Perthame is particularly useful:

Theorem 3.1 (Lions and Perthame).

Let $f_{0} \geq 0, f_{0} \in L^{1}\left(\mathbb{R}^{3} \times \mathbb{R}^{3}\right) \cap L^{\infty}\left(\mathbb{R}^{3} \times \mathbb{R}^{3}\right)$ satisfy

$$
\int|p|^{m} f_{0}(q, p) \mathrm{d} q \mathrm{~d} p<+\infty,
$$

for all $m<m_{0}$ and some $m_{0}>3$.

a) Then, the Vlasov-Poisson system defined by equations (1-3) has a continuous, bounded solution $f(t, \cdot, \cdot) \in C\left(\mathbb{R}^{+} ; L^{p}\left(\mathbb{R}^{3} \times \mathbb{R}^{3}\right)\right) \cap$ $L^{\infty}\left(\mathbb{R}^{+} ; L^{\infty}\left(\mathbb{R}^{3} \times \mathbb{R}^{3}\right)\right)$ for $1 \leq p<\infty$ satisfying

$$
\sup _{t \in[0, T]} \int|p|^{m} f(t, q, p) \mathrm{d} p \mathrm{~d} p<+\infty,
$$

for all $T<\infty, m<m_{0}$.

b) If, in fact, $m_{0}>6$ and we assume that $f_{0}$ satisfies

$$
\begin{array}{r}
\operatorname{esssup}\left\{f_{0}\left(q^{\prime}+p t, p^{\prime}\right):\left|q-q^{\prime}\right| \leq R t^{2},\left|p-p^{\prime}\right|<R t\right\} \\
\in L^{\infty}\left((0, T) \times \mathbb{R}_{q}^{3} ; L^{1}\left(\mathbb{R}_{p}^{3}\right)\right)
\end{array}
$$

for all $R>0$ and $T>0$, then

$$
\sup _{t \in[0, T]}\left\|\rho_{t}(q)\right\|_{\infty}<+\infty, \forall T \in(0,+\infty) .
$$

Under the assumption of part $b$ ) of the theorem, the uniqueness result of Loeper, $2006[13$ then shows that for any $T>0$, said $f$ is the unique solution in the set of bounded, positive measures on $[0, T) \times \mathbb{R}^{6}$ satisfying $\left.f\right|_{t=0}=f_{0}$ in the sense of distributions. Moreover, it is known that as long as the charge density is bounded, solutions with smooth initial data remain smooth (see e.g. in [7]).

As Lions and Perthame remark - and as one can verify by following their proof - part $b$ ) of the theorem actually yields a bound on the charge density that is uniform in $N$ if one considers a sequence of regularized time-evolutions as (for instance) in (10). We will note this important fact in the following lemma.

Lemma 3.2. Let $f_{0} \in L^{1}\left(\mathbb{R}^{3} \times \mathbb{R}^{3}\right) \cap L^{\infty}\left(\mathbb{R}^{3} \times \mathbb{R}^{3}\right)$ and $f_{t}^{N}$ be the solution of the regularized Vlasov-Poisson equation (10) (with corresponding cut-off) and initial datum $f^{N}(0, \cdot, \cdot)=f_{0}$. If $f_{0}$ satisfies 
assumption (20) of the above theorem, there exists a constant $C_{\rho}>0$ such that

$$
\left\|\rho_{t}^{N}\right\|_{\infty}+\left\|\rho_{t}^{N}\right\|_{1} \leq C_{\rho}, \forall N \in \mathbb{N} \cup\{\infty\}, \forall t>0,
$$

where $\rho_{t}^{N}=\rho\left[f_{t}^{N}\right]$ and, with a little abuse of notaiton, $\rho_{t}^{\infty}=\rho\left[f_{t}\right]$.

Since condition (20) is rather abstract, we want to state a more intuitive sufficient criterion.

Lemma 3.3. Let $f_{0} \in L^{1}\left(\mathbb{R}^{3} \times \mathbb{R}^{3}\right) \cap L^{\infty}\left(\mathbb{R}^{3} \times \mathbb{R}^{3}\right), f \geq 0$. Suppose there exist functions $\rho \in L^{\infty}\left(\mathbb{R}^{3}\right)$ and $\vartheta(|p|) \in L^{1}\left(\mathbb{R}^{3}\right)$ with $\vartheta$ monotonously decreasing and an $S>0$ such that for all $|p|>S$

$$
f_{0}(q, p) \leq \rho(q) \vartheta(|p|) .
$$

Then $f_{0}$ satisfies assumption (20). Special cases:

- $f_{0}$ has compact support in the p-variables.

- $f_{0}$ is a thermal state of the form $\rho(q) e^{-\beta p^{2}}$ with $\|\rho\|_{\infty}<\infty, \beta>0$.

Proof. For given $R, t>0$ we have to consider the function

$$
\tilde{f}(t, q, p):=\operatorname{esssup}\left\{f_{0}\left(q^{\prime}+p t, p^{\prime}\right):\left|q-q^{\prime}\right| \leq R t^{2},\left|p-p^{\prime}\right|<R t\right\} .
$$

Choosing $R^{\prime}>S+R T$, we have

$$
\begin{aligned}
& \int_{\mathbb{R}^{3}} \tilde{f}(t, q, p) \mathrm{d}^{3} p=\int_{|p| \leq R^{\prime}}+\int_{|p|>R^{\prime}} \tilde{f}(t, q, p) \mathrm{d}^{3} p \\
\leq & \frac{4}{3} \pi R^{\prime 3}\|\tilde{f}(t, \cdot, \cdot)\|_{\infty}+\|\rho\|_{\infty} \int \sup _{\left|p-p^{\prime}\right|<R t} \vartheta\left(\left|p^{\prime}\right|\right) \mathrm{d}^{3} p \\
\leq & \frac{4}{3} \pi R^{\prime 3}\left\|f_{0}\right\|_{\infty}+\|\rho\|_{\infty} \int \vartheta(|p|-R t) \mathrm{d}^{3} p \\
\leq & C\left\|f_{0}\right\|_{\infty}+\|\rho\|_{\infty}\|\vartheta\|_{1}<\infty,
\end{aligned}
$$

where in the second to last line we used the monotonicity of $\vartheta(|p|)$ and the fact that $\|\tilde{f}\|_{\infty}=\left\|f_{0}\right\|_{\infty}$.

One crucial consequence of the bounded density is that the mean field force remains bounded, as well.

Lemma 3.4. Let $k$ be the Coulomb kernel, and $\rho \in L^{1} \cap L^{\infty}\left(\mathbb{R}^{3} ; \mathbb{R}^{+}\right)$. Then there exists $C>0$ such that

$$
\|k * \rho\|_{\infty} \leq C\|\rho\|_{1}^{1 / 3}\|\rho\|_{\infty}^{2 / 3} .
$$

Proof. For $R>0$, we compute:

$$
\begin{aligned}
\|k * \rho\|_{\infty} & \leq\left\|\int_{|y|<R} k(y) \rho(x-y) \mathrm{d}^{3} y\right\|_{\infty}+\left\|\int_{|y| \geq R} k(y) \rho(x-y) \mathrm{d}^{3} y\right\|_{\infty} \\
& \leq\|\rho\|_{\infty} \int_{|y|<R} \frac{1}{|y|^{2}} \mathrm{~d}^{3} y+R^{-2}\|\rho\|_{1}=4 \pi R\|\rho\|_{\infty}+R^{-2}\|\rho\|_{1} .
\end{aligned}
$$

This last expression is optimized by setting $R=(4 \pi)^{-1 / 3}\|\rho\|_{\infty}^{-1 / 3}\|\rho\|_{1}^{1 / 2}$, which yields $\|k * \rho\|_{\infty} \leq 2(4 \pi)^{2 / 3}\|\rho\|_{1}^{1 / 3}\|\rho\|_{\infty}^{2 / 3}$. 


\section{Statement of the results}

In the following, all probabilities and expectation values are meant with respect to the product measure given at a certain time by $f_{t}^{N}$. That is, for any random variable $H: \mathbb{R}^{6 N} \rightarrow \mathbb{R}$ and any element $A$ of the Borel algebra

$$
\begin{aligned}
\mathbb{P}_{t}^{N}(H \in A) & =\int_{H^{-1}(A)} \prod_{j=1}^{N} f_{t}^{N}\left(z_{j}\right) d Z \\
\mathbb{E}_{t}^{N}(H) & =\int_{\mathbb{R}^{6 N}} H(Z) \prod_{j=1}^{N} f_{t}^{N}\left(z_{j}\right) d Z .
\end{aligned}
$$

Note that since ${ }^{N} \Phi_{t, s}$ leaves the measure invariant,

$$
\begin{aligned}
\mathbb{E}_{s}^{N}\left(H \circ{ }^{N} \Phi_{t, s}\right) & =\int_{\mathbb{R}^{6 N}} H\left({ }^{N} \Phi_{t, s}(Z)\right) \prod_{j=1}^{N} f_{s}^{N}\left(z_{j}\right) d Z \\
& =\int_{\mathbb{R}^{6 N}} H(Z) \prod_{j=1}^{N} f_{s}^{N}\left(\varphi_{s, t}^{N}\left(z_{j}\right)\right) d Z \\
& =\int_{\mathbb{R}^{6 N}} H(Z) \prod_{j=1}^{N} f_{t}^{N}\left(z_{j}\right) d Z=\mathbb{E}_{t}^{N}(H) .
\end{aligned}
$$

In particular:

$$
\mathbb{P}_{t}^{N}(Z \in A)=\mathbb{P}_{0}^{N}\left({ }^{N} \Phi_{t, 0}(Z) \in A\right) .
$$

We will often omit the index $N$ for $\mathbb{P}_{0}$ and $\mathbb{E}_{0}$ defined with respect to the product measure $\otimes^{N} f_{0}$.

To quantify the convergence of probability measures, we will use the Wasserstein distances (also known as Monge-Kantorivich-Rubinstein distances). In the context of kinetic equations, they were first introduced by Dobrushin in [3]. We shall briefly recall the definition and some basic properties. For further details, we refer the reader to the book of Villani [20, Ch. 6].

Definition 4.1. Let $\mathcal{P}\left(\mathbb{R}^{n}\right)$ be the set of probability measures on $\mathbb{R}^{n}$. For given $\mu, \nu \in \mathcal{P}\left(\mathbb{R}^{n}\right)$, let $\Pi(\mu, \nu)$ be the set of all probability measures $\mathbb{R}^{n} \times \mathbb{R}^{n}$ with marginal $\mu$ and $\nu$, respectively. Then, for $p \in[1,+\infty)$, the $p^{\prime}$ th Wasserstein distance on $\mathcal{P}\left(\mathbb{R}^{n}\right)$ is defined by

$$
W_{p}(\mu, \nu):=\inf _{\pi \in \Pi(\mu, \nu)}\left(\int_{\mathbb{R}^{n} \times \mathbb{R}^{n}}|x-y|^{p} \mathrm{~d} \pi(x, y)\right)^{1 / p} .
$$

Convergence in Wasserstein distance implies, in particular, weak convergence in $\mathcal{P}\left(\mathbb{R}^{n}\right)$, i.e.

$$
\int \Phi(x) \mathrm{d} \mu_{k}(x) \rightarrow \int \Phi(x) \mathrm{d} \mu(x), \quad k \rightarrow \infty,
$$


for all bonded, continuous functions $\Phi$. Moreover, convergence in $W_{p}$ implies convergence of the first $p$ moments. $W_{p}$ satisfies all properties of a metric on $\mathcal{P}\left(\mathbb{R}^{n}\right)$, except that it may take the value $+\infty$.

The most common version is the first Wasserstein distance, for which we have the Kantorovich-Rubinstein duality:

$$
W_{1}(\mu, \nu)=\sup _{\|g\|_{L i p} \leq 1}\left\{\int g(x) \mathrm{d} \mu(x)-\int g(x) \mathrm{d} \nu(x)\right\},
$$

where $\|g\|_{L i p}:=\sup _{x, y} \frac{g(x)-g(y)}{|x-y|}$, for $g: \mathbb{R}^{n} \rightarrow \mathbb{R}$. We will also consider the infinite Wasserstein distance defined by

$$
W_{\infty}(\mu, \nu)=\inf \{\pi-\operatorname{esssup}|x-y|: \pi \in \Pi(\mu, \nu)\} .
$$

We can now state our precise results in the following theorem.

Theorem 4.2 (Molecular chaos). Let $f_{0} \in L^{\infty}\left(\mathbb{R}^{3} \times \mathbb{R}^{3}\right)$ a probability measure satisfying the assumptions of Theorem 3.1 a) and b) and $f^{N}$ the unique solution of the regularized Vlasov-Poisson equation (10) with initial datum $f_{0}$. For $0<\delta<\frac{1}{3}$ let ${ }^{N} \Psi_{t, s}$ be the $N$-particle flow solving (5) with cut-off width $N^{-\delta}$ and let ${ }^{N} \Phi_{t, s}$ be the $N$-particle mean field flow induced by $f^{N}$ as defined in (14). Then, for any $T>0$, there exists a constant $C_{0}$ depending on $\sup _{N \in \mathbb{N}}\left\|\rho\left[f^{N}\right]\right\|_{L^{\infty}\left([0, T] \times \mathbb{R}^{3}\right)}$ such that for any $\beta>0$ there exists a constant $C_{\beta}$ such that for all $N \geq N_{0}:=e^{\left(\frac{C_{0} T+1}{1-3 \delta}\right)^{2}}$

$$
\mathbb{P}_{0}\left[\exists t \in[0, T]:\left|{ }^{N} \Psi_{t, 0}(Z)-{ }^{N} \Phi_{t, 0}(Z)\right|_{\infty} \geq N^{-\delta}\right] \leq \frac{T C_{\beta}}{N^{\beta}},
$$

where $|\cdot|_{\infty}$ denotes the maximum-norm on $\mathbb{R}^{6 N}$.

This result implies molecular chaos in the following sense:

Corollary 4.3. Let $F_{0}^{N}:=\otimes^{N} f_{0}$ and $F_{t}^{N}:={ }^{N} \Psi_{t, 0} \# F_{0}$ the $N$-particle distribution evolving with the microscopic flow (7). Then the $k$-particle marginal

$$
{ }^{(k)} F_{t}^{N}\left(z_{1}, \ldots, z_{k}\right):=\int F_{t}^{N}(Z) \mathrm{d}^{6} z_{k+1} \ldots \mathrm{d}^{6} z_{N}
$$

converges weakly to $\otimes^{k} f_{t}$ as $N \rightarrow \infty$ for all $k \in \mathbb{N}$, where $f_{t}$ is the unique solution of the Vlasov-Poisson equation (11) with $\left.f^{N}\right|_{t=0}=f_{0}$. More precisely, under the assumptions of the previous theorem, we get a constant $C>0$ such that for all $N \geq N_{0}$

$$
W_{1}\left({ }^{(k)} F_{t}^{N}, \otimes^{k} f_{t}\right) \leq k e^{T C \sqrt{\log (N)}} N^{-\delta}, \forall 0 \leq t \leq T .
$$

Proof. For fixed $0 \leq t \leq T$, let $\mathcal{A} \subset \mathbb{R}^{6 N}$ be the set defined by $Z \in$ $\mathcal{A} \Longleftrightarrow\left|{ }^{N} \Psi_{t, 0}(Z)-{ }^{N} \Phi_{t, 0}(Z)\right|_{\infty}<N^{-\delta}$. Hence, according to the previous theorem, $\mathbb{P}_{0}\left(\mathcal{A}^{c}\right) \leq \frac{T C_{\beta}}{N^{\beta}}$ for sufficiently large $N$. In view of 
the Kantorovich-Rubinstein duality (28), we have:

$$
\begin{aligned}
W_{1}\left({ }^{(k)} F_{t}^{N}, \otimes^{k} f_{t}\right) \\
=\sup _{\|g\|_{L i p}=1}\left|\int\left({ }^{(k)} F_{t}^{N}-\otimes^{k} f_{t}\right) g\left(z_{1}, \ldots, z_{k}\right) \mathrm{d}^{6} z_{1} \ldots \mathrm{d}^{6} z_{k}\right| \\
=\sup _{\|g\|_{L i p}=1}\left|\int\left(F_{t}^{N}(Z)-\otimes^{N} f_{t}(Z)\right) g\left(z_{1}, \ldots, z_{k}\right) \mathrm{d}^{6} z_{1} \ldots \mathrm{d}^{6} z_{k} \ldots \mathrm{d}^{6} z_{N}\right| \\
=\sup _{\|g\|_{L i p}=1}\left|\int\left(\Psi_{t, 0} \# F_{0}^{N}(Z)-\Phi_{t, 0} \# F_{0}^{N}(Z)\right) g\left(z_{1}, \ldots, z_{k}\right) \mathrm{d}^{6 N} Z\right|
\end{aligned}
$$

Introducing the projection $P_{k}: \mathbb{R}^{N} \rightarrow \mathbb{R}^{k},\left(z_{1}, \ldots, z_{N}\right) \mapsto\left(z_{1}, \ldots, z_{k}\right)$, this can be further rewritten as

$$
\begin{aligned}
& W_{1}\left({ }^{(k)} F_{t}^{N}, \otimes^{k} f_{t}\right) \\
& =\sup _{\|g\|_{L i p}=1}\left|\int F_{0}^{N}(Z)\left(g\left(P_{k} \Psi_{t, 0}(Z)\right)-g\left(P_{k} \Phi_{t, 0}(Z)\right)\right) \mathrm{d}^{6 N} Z\right| \\
& =\sup _{\|g\|_{L i p}=1}\left|\int_{\mathcal{A}^{c}} F_{0}^{N}(Z)\left(g\left(P_{k} \Psi_{t, 0}(Z)\right)-g\left(P_{k} \Phi_{t, 0}(Z)\right)\right) \mathrm{d}^{6 N} Z\right| \\
& +\sup _{\|g\|_{L i p}=1}\left|\int_{\mathcal{A}} F_{0}^{N}(Z)\left(g\left(P_{k} \Psi_{t, 0}(Z)\right)-g\left(P_{k} \Phi_{t, 0}(Z)\right)\right) \mathrm{d}^{6 N} Z\right| .
\end{aligned}
$$

Using that all test-functions are Lipschitz with $\|g\|_{\text {Lip }}=1$, we have (33) $\leq\left.\mathbb{P}_{0}\left(\mathcal{A}^{c}\right)\left\|F_{0}^{N}\right\|_{\infty}\right|^{N} \Psi_{t, 0}(Z)-{ }^{N} \Phi_{t, 0}(Z) \mid$, with $\left\|F_{0}^{N}\right\|_{\infty}=\left(\left\|f_{0}\right\|_{\infty}\right)^{N}$. Recalling that $\left|{ }^{N} \Psi_{0,0}(Z)-{ }^{N} \Phi_{0,0}(Z)\right|_{\infty}=0$, we have

$$
\begin{aligned}
\left|{ }^{N} \Psi_{t, 0}^{2}(Z)-{ }^{N} \Phi_{t, 0}^{2}(Z)\right|_{\infty} & \leq \int_{0}^{t}\left|K_{\delta}^{N}\left(\Psi_{s, 0}^{1}(Z)\right)-\bar{K}\left(\Phi_{s, 0}^{1}(Z)\right)\right|_{\infty} \mathrm{d} s, \\
\left|{ }^{N} \Psi_{t, 0}^{1}(Z)-{ }^{N} \Phi_{t, 0}^{1}(Z)\right|_{\infty} & \leq \int_{0}^{t}\left|{ }^{N} \Psi_{s, 0}^{2}(Z)-{ }^{N} \Phi_{s, 0}^{2}(Z)\right|_{\infty} \mathrm{d} s .
\end{aligned}
$$

The mean field force $\bar{K}$ is of order 1 (Lemma 3.4), while the microscopic force $K_{\delta}^{N}$ is bounded by $N^{2 \delta}$. Hence, there exists a constant $C^{\prime}>0$ such that $\left.\right|^{N} \Psi_{t, 0}^{2}(Z)-\left.{ }^{N} \Phi_{t, 0}^{2}(Z)\right|_{\infty} \leq T C^{\prime} N^{2 \delta}$ and consequently $\left|{ }^{N} \Psi_{t, 0}^{1}(Z)-{ }^{N} \Phi_{t, 0}^{1}(Z)\right|_{\infty} \leq T^{2} C^{\prime} N^{2 \delta}$ for all $t \leq T$. Choosing $\beta:=3 \delta$ in (30) we thus get another constant $C^{\prime \prime}$ such that

$$
\text { (33) } \leq C^{\prime \prime} \max \left\{T^{2}, T^{3}\right\} N^{-\delta}, \forall 0 \leq t \leq T \text {. }
$$

On the other hand, for $Z \in \mathcal{A}$, we have for any $g$ with $\|g\|_{L i p}=1$,

$$
\left|g\left(P_{k}{ }^{N} \Psi_{t, 0}(Z)\right)-g\left(P_{k}{ }^{N} \Phi_{t, 0}(Z)\right)\right| \leq\left.\right|^{N} \Psi_{t, 0}(Z)-\left.{ }^{N} \Phi_{t, 0}(Z)\right|_{\infty} \leq N^{-\delta}
$$

for all $t \leq T$ and thus (34) $\leq N^{-\delta}$. Together with (35), we get a constant $C^{\prime \prime \prime}$ such that

$$
W_{1}\left({ }^{(k)} F_{t}^{N}, \otimes^{k} f_{t}^{N}\right) \leq C^{\prime \prime \prime}\left(1+T^{3}\right) N^{-\delta}, \forall 0 \leq t \leq T .
$$


Finally, we will prove in Proposition 9.1 that

$$
W_{1}\left(f_{t}^{N}, f_{t}\right) \leq N^{-\delta} e^{t 2 C_{0} \sqrt{\log N}}, \forall t \leq T
$$

where $C_{0}$ depends only on $f_{0}$ and $T$. Putting everything together and using $W_{1}\left({ }^{(k)} F_{t}^{N}, \otimes^{k} f_{t}\right) \leq W_{1}\left({ }^{(k)} F_{t}^{N}, \otimes^{k} f_{t}^{N}\right)+W_{1}\left(\otimes^{k} f_{t}^{N}, \otimes^{k} f_{t}\right)$ the statement follows.

It is a classical result in probability theory (see e.g. [9], [5], [19, Prop.2.2]) that molecular chaos in the sense of the previous corollary implies convergence in law of the empirical distribution $\mu_{t}^{N}[Z]:=\mu_{0}^{N}\left[\Psi_{t, 0}(Z)\right]$ to the constant variable $f_{t}$. However, under additional assumptions on the decay of $f_{0}$, we can obtain the following quantitative result:

Theorem 4.4 (Particle approximation of the Vlasov-Poisson system). Let $f_{0} \in L^{\infty}\left(\mathbb{R}^{3} \times \mathbb{R}^{3}\right)$ a probability measure satisfying the assumptions of Theorem 3.1 a) and b). For $0<\delta<\frac{1}{3}$, let ${ }^{N} \Psi_{t, s}$ be the $N$-particle flow solving (5) with cut-off width $N^{-\delta}$. Let $p \in[1, \infty)$ and assume that, in addition, there exists $k>2 p$ such that $\int_{\mathbb{R}^{6}}|z|^{k} \mathrm{~d} f_{0}(z)<+\infty$. Then, the empirical density $\mu_{t}^{N}[Z]:=\mu_{0}^{N}\left[\Psi_{t, 0}(Z)\right]$ converges to the solution of the Vlasov-Poisson equation in the following sense:

For any $T>0$ and $\gamma<\min \left\{\frac{1}{6}, \frac{1}{2 p}, \delta\right\}$, there exists a constant $C_{0}$ depending on $f_{0}$ and $T$ and constants $c, C_{1}, C_{2}$ depending on $k, p, \gamma$ such that for all $N \geq N_{1}:=e^{\left(\frac{2\left(C_{0} T+1\right)}{1-3 \delta}\right)^{2}}$

$$
\begin{array}{r}
\mathbb{P}_{0}\left[\exists t \in[0, T]: W_{p}\left(\mu_{t}^{N}[Z], f_{t}\right)>N^{-\gamma+1-3 \delta}\right] \\
\leq C_{1} e^{-c N^{1-(6 \vee 2 p) \gamma}}+T C_{2} N^{1-\frac{k}{2 p}}
\end{array}
$$

where $f$ is the unique solution of the Vlasov-Poisson system on $[0, T]$ with $\left.f\right|_{t=0}=f_{0}$ and $6 \vee 2 p:=\max \{6,2 p\}$.

The proofs of the theorems will be given in Sections 8 and 9 .

\section{Remarks 4.5.}

1) Our results allow to choose the width of the cut-off arbitrary close to $N^{-1 / 3}$ which corresponds to the scale of the typical distance between a particle and its nearest neighbor.

2) The results can be straightforwardly generalized to include external forces or noise, provided that the regularity of solutions to the corresponding mean field equation remains as assumed.

3) All dynamical estimates required for Theorem 4.4 would hold directly for the infinite Wasserstein distance. However, there are no concentration estimates available in terms of $W_{\infty}$ to establish a good approximation of the initial $f_{0}$ by the empirical measure $\mu_{0}^{N}$. 


\section{A new measure of chaos}

The strategy of proof is to control the deviation of the microscopic time evolution from the mean field time evolution in terms of the following $N$-dependent quantity:

Definition 5.1. Let ${ }^{N} \Phi_{t, 0}$ the mean field flow defined in (14) and ${ }^{N} \Psi_{t, 0}$ the microscopic flow defined in (6). We denote by ${ }^{N} \Phi_{t, 0}^{1}=$ $\left(q_{i}(t)\right)_{1 \leq i \leq N}$ and ${ }^{N} \Phi_{t, 0}^{2}=\left(p_{i}(t)\right)_{1 \leq i \leq N}$ the projection onto the spatial, respectively the momentum coordinates.

Consider the quantity $\Delta_{Z, t}^{N}$ defined as

$$
\begin{array}{r}
\Delta_{Z, t}^{N}=\left.\sqrt{\log (N)}\right|^{N} \Psi_{t, 0}^{1}(Z)-\left.{ }^{N} \Phi_{t, 0}^{1}(Z)\right|_{\infty} \\
+\left.\right|^{N} \Psi_{t, 0}^{2}(Z)-\left.{ }^{N} \Phi_{t, 0}^{2}(Z)\right|_{\infty},
\end{array}
$$

where $|Z|_{\infty}=\max \left\{\left|z_{i}\right|: 1 \leq i \leq 3 N\right\}$ is the maximum-norm on $\mathbb{R}^{3 N}$. In view of Theorem 4.2, our aim is to show that for any $\epsilon>0$ :

$$
\mathbb{P}_{0}\left[\sup _{0 \leq s \leq T}\left\{\Delta_{Z, s}^{N}\right\} \geq N^{-\delta}\right] \rightarrow 0
$$

faster than any inverse power of $N$. This will be done by introducing for $\lambda>0$ and $N \in \mathbb{N}$ the stochastic process $J_{t}^{N, \lambda}(Z)$ given by

$$
J_{t}^{N, \lambda}(Z):=\min \left\{1, \sup _{0 \leq s \leq t}\left\{e^{\lambda \sqrt{\log (N)}(T-s)}\left(N^{\delta} \Delta_{Z, s}^{N}+N^{3 \delta-1}\right)\right\}\right\}
$$

and controlling the evolution of $\mathbb{E}_{0}^{N}\left(J_{t}^{N, \lambda}\right)$.

The crucial innovation with respect to [1] is that distances in spatial and momentum coordinates are weighted differently by a $N$-dependent factor (here: $\sqrt{\log (N)})$, exploiting the second-order nature of the dynamics. Moreover, the quantity $J_{t}^{N, \lambda}$ has been redefined in order to optimize the rate of convergence.

The relevance of (39) for the proof of Theorem 4.4 is grounded in the following observation:

Lemma 5.2. For $X=\left(x_{1}, \ldots, x_{N}\right) \in \mathbb{R}^{6 N}$ let $\mu_{0}^{N}[X]:=\frac{1}{N} \sum_{i=1}^{N} \delta_{x_{i}} \in$ $\mathcal{P}\left(\mathbb{R}^{6 N}\right)$. Then we have for all $p \in[1, \infty]$ :

$$
W_{p}\left(\mu_{0}^{N}[X], \mu_{0}^{N}[Y]\right) \leq|X-Y|_{\infty} .
$$

This implies, in particular, for any $\xi>0$

$$
\begin{array}{r}
\mathbb{P}_{0}\left[\sup _{0 \leq s \leq t} W_{p}\left(\mu_{0}^{N}\left[\Psi_{s, 0}(Z)\right], \mu_{0}^{N}\left[\Phi_{s, 0}(Z)\right]\right) \geq \xi\right] \\
\leq \mathbb{P}_{0}\left[\sup _{0 \leq s \leq T}\left\{\Delta_{Z, s}^{N}\right\} \geq \xi\right] .
\end{array}
$$


Proof of the Lemma. Since $W_{p} \leq W_{q}$ for $p \leq q$, it suffices to consider the infinite Wasserstein distance defined in (29).

We then observe that $\pi_{0}=\sum_{i=1}^{N} \delta_{x_{i}} \delta_{y_{i}} \in \Pi\left(\mu_{0}^{N}[Z], \mu_{0}^{N}[Y]\right)$ with $\pi_{0}-$ $\operatorname{esssup}|x-y|=\max _{1 \leq i \leq N}\left|x_{i}-y_{i}\right|=|X-Y|_{\infty}$.

In total, we will split our approximation result for the Vlasov-Poisson equation into

$$
\begin{aligned}
W_{p}\left(\mu_{t}^{N}[Z], f_{t}\right) & \leq W_{p}\left(\mu_{0}^{N}\left[\Psi_{t, 0}(Z)\right], \mu_{0}^{N}\left[\Phi_{t, 0}(Z)\right]\right) \\
& +W_{p}\left(\mu_{0}^{N}\left[\Phi_{t, 0}(Z)\right], f_{t}^{N}\right) \\
& +W_{p}\left(f_{t}^{N}, f_{t}\right)
\end{aligned}
$$

The first term (42) is the interesting one, concerning the difference between microscopic time-evolution and mean field time-evolution. It will be controlled in terms of $\mathbb{E}_{0}\left(J_{t}^{N, \lambda}\right)$ and shown to converge in probability faster than any inverse power of $N$.

The second term $W_{p}\left(\mu_{0}^{N}\left[\Phi_{t, 0}(Z)\right], f_{t}\right)=W_{p}\left(\varphi_{t, 0}^{N} \# \mu_{0}^{N}[Z], \varphi_{t, 0}^{N} \# f_{0}\right)$ concerns the sampling of the mean field dynamics by discrete particle trajectories. We will use a recent large deviation estimate of Fournier and Guillin [4 to determine the typical rates of convergence for the initial distribution and then control the growth of (43) by a Gronwall estimate.

Convergence of (44) is a purely deterministic result: solutions of the regularized Vlasov-Poisson equation (10) approximate solutions of the proper Vlasov-Poisson equation (1) as the width of the cut-off goes to zero.

The central idea of our strategy is thus to first sample the (regularized) mean field dynamics along trajectories with random initial conditions, i.e. approximate $f_{t}^{N}$ by $\mu_{0}^{N}\left[\Phi_{t, 0}(Z)\right]$, and then control the difference between mean field trajectories and the "true" microscopic trajectories in terms of the expectation $\mathbb{E}_{0}^{N}\left(J_{t}^{N, \lambda}\right)$. This approach has several important virtues:

1. The method is designed for stochastic initial conditions, thus allowing for law-of-large number estimates that turn out to be very powerful. (Note that the particles evolving with the mean field flow remain statistically independent at all times.)

2. The metric $\left.\right|^{N} \Psi_{t, 0}(Z)-\left.{ }^{N} \Phi_{t, 0}(Z)\right|_{\infty}$ is much stronger than usual weak distances between probability measures, thus allowing for better stability estimates.

3. Since $\frac{\mathrm{d}}{\mathrm{d} t} J_{t}^{N, \lambda}(Z) \leq 0$ if $\sup _{0 \leq s \leq t}\left|{ }^{N} \Psi_{s, 0}(Z)-{ }^{N} \Phi_{s, 0}(Z)\right|_{\infty} \geq N^{-\delta}$ we only have to consider situations in which mean field trajectories and microscopic trajectories are still close together.

4. Exploiting the second-order nature of the dynamics, we weigh distances in $x$-space and momentum space differently, with an 
$N$-dependent factor $\sqrt{\log (N)}$. As we compare microscopic trajectories to characteristic curves of the mean field equation, the growth the spatial distance is trivially bounded by the difference of the respective momenta. The idea is thus to be a little more strict on deviations in space, so to speak, and use this to obtain better control on fluctuations of the force.

\section{$6 \quad$ Local Lipschitz bound}

If all forces were Lipschitz continuous with a Lipschitz constant $L$ independent of $N$, we could easily conclude that $\frac{\mathrm{d}}{\mathrm{d} t}\left|{ }^{N} \Psi_{t, 0}(Z)-{ }^{N} \Phi_{t, 0}(Z)\right|_{\infty} \leq$ $\left.(1+L)\right|^{N} \Psi_{t, 0}(Z)-\left.{ }^{N} \Phi_{t, 0}(Z)\right|_{\infty}$, from which the desired convergence readily follows. However, the forces considered here become singular in the limit $N \rightarrow \infty$ and hence do not satisfy a uniform Lipschitz bound. Nevertheless, we observe that, for the mean field force $k_{\delta}^{N} * \rho_{t}^{N}$, the global Lipschitz constant $\left\|k^{N} * \rho_{t}^{N}\right\|_{\text {Lip }}$ diverges only logarithmically as the cut-off is lifted with increasing $N$. Due to the pre-factor $\sqrt{\log (N)}$ in Definition 5.1, the particular anisotropic scaling of our metric will allow us to "trade" part of this divergence for a tighter control on spatial fluctuations. This will suffice to establish the desired convergence, using the fact that $e^{\sqrt{\log (N)}}=N^{\frac{1}{\sqrt{\log (N)}}}$ grows slower than $N^{\epsilon}$ for any $\epsilon>0$. (C.f. also [11] where we have implemented the same idea).

We summarize our first observation in the following Lemma.

Lemma 6.1. Let $0<\gamma<1$ and assume that $l: \mathbb{R}^{3} \rightarrow \mathbb{R}^{k}$ satisfies

$$
|l(q)| \leq c \cdot \min \left\{N^{3 \gamma},|q|^{-3}\right\}
$$

for some $c>0$. Then there exists a constant $C_{l}>0$ such that

$$
\left\|l * \rho_{t}(x)\right\|_{\infty} \leq C_{l} \max \{1, \sqrt{\log (N)}\}\left(\left\|\rho_{t}\right\|_{1}+\left\|\rho_{t}\right\|_{\infty}\right) .
$$

Proof. We estimate

$$
\begin{aligned}
\left\|l * \rho_{t}(x)\right\|_{\infty} & =\left\|\int l(x-y) \rho_{t}(y) \mathrm{d}^{3} y\right\|_{\infty} \\
& \leq\left\|\int_{|x-y|<N^{-\gamma}} l(x-y) \rho_{t}(y) \mathrm{d}^{3} y\right\|_{\infty} \\
& +\left\|\int_{N-\gamma<|x-y|<1} l(x-y) \rho_{t}(y) \mathrm{d}^{3} y\right\|_{\infty} \\
& +\left\|\int_{|x-y|>1} l(x-y) \rho_{t}(y) \mathrm{d}^{3} y\right\|_{\infty} .
\end{aligned}
$$

The first term is bounded by

$$
\left\|\int_{|x-y|<N^{-\gamma}} l(x-y) \rho_{t}(y) \mathrm{d}^{3} y\right\|_{\infty} \leq\left\|\rho_{t}\right\|_{\infty} N^{3 \gamma}\left|\mathrm{B}\left(N^{-\gamma}\right)\right| \leq \frac{4}{3} \pi\left\|\rho_{t}\right\|_{\infty},
$$


where $\mathrm{B}(r)$ denotes the ball with radius $r$. The last term is bounded by

$$
\left\|\int_{|x-y|>1} l(x-y) \rho_{t}(y) \mathrm{d}^{3} y\right\|_{\infty} \leq c\left\|\rho_{t}\right\|_{1} .
$$

Finally, the second term yields

$$
\begin{aligned}
\left\|\int_{N^{-\gamma}<|x-y|<1} g(x-y) \rho_{t}(y) \mathrm{d}^{3} y\right\|_{\infty} & \leq\left\|\rho_{t}\right\|_{\infty} \int_{N^{-\gamma<|y|<1}} \frac{c}{|y|^{3}} \mathrm{~d}^{3} y \\
& \leq 4 \pi c \gamma\left\|\rho_{t}\right\|_{\infty} \log (N) .
\end{aligned}
$$

One immediate application of the Lemma is to $l(q)=\nabla k_{\delta}^{N}(q)$, showing that a regularized mean field force is Lipschitz continuous with a constant proportional to $\log (N)$. Our aim is now to prove that for typical initial conditions, fluctuations in the microscopic forces can be bound in a similar fashion, as long as ${ }^{N} \Psi_{t, 0}(Z)$ and ${ }^{N} \Phi_{t, 0}(Z)$ are close.

Definition 6.2. Let

$$
l_{\delta}^{N}(q):= \begin{cases}\frac{54}{|q|^{3}} & , \text { if }|q| \geq 3 N^{-\delta} \\ N^{3 \delta} & , \text { else }\end{cases}
$$

and $L: \mathbb{R}^{6 N} \rightarrow \mathbb{R}^{N}$ be defined by $(L(Z))_{i}:=\frac{1}{N} \sum_{j \neq i} l_{\delta}^{N}\left(q_{i}-q_{j}\right)$. Furthermore, we define $\bar{L}_{t}(Z)$ by $\left(\bar{L}_{t}(Z)\right)_{i}:=l_{\delta}^{N} * \rho_{t}^{N}\left(q_{i}\right)=\int l_{\delta}^{N} *_{q}$ $f\left(t, q_{i}, p\right) \mathrm{d}^{3} p$.

Lemma 6.3. For any $\xi \in \mathbb{R}^{3}$ with $|\xi|_{\infty}<2 N^{-\delta}$, it holds that

$$
\left|k_{\delta}^{N}(q)-k_{\delta}^{N}(q+\xi)\right|_{\infty} \leq l_{\delta}^{N}(q)|\xi|_{\infty} .
$$

Proof. First note that by assumption the derivative of $k^{N}$ is bounded by $N^{3 \delta}$, so that (48) holds for $|q|<3 N^{-\delta}$. For $|q| \geq 3 N^{-\delta}$, there exists $s \in[0,1]$ such that $\left|k_{\delta}^{N}(q)-k_{\delta}^{N}(q+\xi)\right| \leq\left|\nabla k_{\delta}^{N}(q+s \xi)\right|_{\infty}|\xi|_{\infty}$, where

$$
\left|\nabla k_{\delta}^{N}(q+s \xi)\right|_{\infty} \leq 2|q+s \xi|^{-3} .
$$

The expression on the right-hand-side takes its greatest value if $\xi$ is antiparallel to $q$ and $s=1$. Hence, we have

$$
\left|k_{\delta}^{N}(q)-k_{\delta}^{N}(q+\xi)\right|_{\infty} \leq 2\left|q\left(1-\frac{|\xi|}{|q|}\right)\right|^{-3}|\xi|_{\infty} .
$$

Since $|q| \geq 3 N^{-\delta}$ and $|\xi|<2 N^{-\delta}$, it follows that $\frac{|\xi|}{|q|}<\frac{2}{3}$. Thus, we get $\left|k_{\delta}^{N}(q)-k_{\delta}^{N}(q+\xi)\right|_{\infty} \leq 2\left(\frac{3}{|q|}\right)^{3}|\xi|_{\infty} \leq \frac{54}{|q|^{3}}|\xi|_{\infty}$. 


\section{$7 \quad$ Law of large numbers}

In order to control the evolution of $\mathbb{E}_{0}\left(J_{t}^{N}\right)$, we will require as an intermediate step that the mean field force (and its derivative) can be approximated by the analogous expressions for the discrete measure $\mu_{0}^{N}\left[\Phi_{t, 0}(Z)\right]$ with random $Z$. The key observation here is that if the $N$-particle configuration evolves with the mean field flow ${ }^{N} \Phi_{t, 0}$, the particles remain statistically independent for all $t$, thus giving rise to a law-of-large-numbers estimate.

Definition 7.1. For any $t>0$ and fixed $\delta<\frac{1}{3}$, we consider the $(N$ and $t$ dependent) sets $\mathcal{A}_{t}, \mathcal{B}_{t}, \mathcal{C}_{t}$ defined by

$$
\begin{aligned}
& Z \in \mathcal{A}_{t} \Longleftrightarrow\left|J_{t}^{N, \lambda}(Z)\right|<1 \\
& Z \in \mathcal{B}_{t} \Longleftrightarrow\left|K_{\delta}^{N}\left(\Phi_{t, 0}(Z)\right)-\bar{K}\left(\Phi_{t, 0}(Z)\right)\right|_{\infty}<N^{-1+2 \delta} \\
& Z \in \mathcal{C}_{t} \Longleftrightarrow\left|L_{\delta}^{N}\left(\Phi_{t, 0}(Z)\right)-\bar{L}\left(\Phi_{t, 0}(Z)\right)\right|_{\infty}<1
\end{aligned}
$$

where $\bar{K}$ is the mean field force (15) and $\bar{L}$ as in Definition 6.2

We now want to show that for any $t$, initial conditions in $\mathcal{B}_{t} \cap \mathcal{C}_{t}$ are typical with respect to the product measure $F_{0}:=\otimes^{N} f_{0}$ on $\mathbb{R}^{6 N}$.

Proposition 7.2. Let $\rho_{t} \in L^{1}\left(\mathbb{R}^{3}\right) \cap L^{\infty}\left(\mathbb{R}^{3}\right)$ with $\left\|\rho_{t}\right\|_{1}=1$ as before. Let $h: \mathbb{R}^{3} \rightarrow \mathbb{R}$ and suppose that for given $\delta>0$ and $N \in \mathbb{N}$ there exists $c>0$ and an exponent $2 \leq \alpha \leq 3$ such that $|h(x)| \leq c \cdot \min \left\{N^{\alpha \delta},|q|^{-\alpha}\right\}, \forall q \in \mathbb{R}^{3}$. Assume furthermore that

$$
\delta<\min \left\{\frac{1-2 \beta}{2 \alpha-3}, \frac{1-\beta}{\alpha}\right\} .
$$

Then there exists for all $\gamma>0$ a constant $C_{\kappa}>0$ such that

$$
\mathbb{P}_{t}\left[\sup _{1 \leq i \leq N}\left|\frac{1}{N} \sum_{j \neq i}^{N} h\left(q_{i}-q_{j}\right)-h * \rho_{t}\left(q_{i}\right)\right| \geq N^{-\beta}\right] \leq \frac{C_{\kappa}}{N^{\kappa}} .
$$

Proof. Let

$$
D_{i}:=\left\{Z \in \mathbb{R}^{6}:\left|\frac{1}{N} \sum_{j \neq i}^{N} h\left(q_{i}-q_{j}\right)-h * \rho_{t}\left(q_{i}\right)\right| \geq N^{-\beta}\right\}
$$

and $D:=\bigcup_{i=1}^{N} D_{i}$. Then $\mathbb{P}(D) \leq \sum_{i=1}^{N} \mathbb{P}\left(D_{i}\right)=N \mathbb{P}\left(D_{1}\right)$.

By Markov's inequality, we have for every $M \in \mathbb{N}$ :

$$
\begin{aligned}
\mathbb{P}_{t}\left(D_{1}\right) & \leq \mathbb{E}_{t}\left[N^{2 M \beta}\left|\frac{1}{N} \sum_{j=1}^{N} h\left(q_{1}-q_{j}\right)-h * \rho_{t}\left(q_{1}\right)\right|^{2 M}\right] \\
& =\frac{1}{N^{2 M(1-\beta)}} \mathbb{E}\left[\left(\sum_{j=1}^{N}\left(h\left(q_{1}-q_{j}\right)-h * \rho_{t}\left(q_{i}\right)\right)\right)^{2 M}\right] .
\end{aligned}
$$


Let $\mathcal{M}:=\left\{\mathbf{k} \in \mathbb{N}_{0}^{N}|| \mathbf{k} \mid=2 M\right\}$ the set of multiindices $\mathbf{k}=\left(k_{1}, \ldots, k_{N}\right)$ with $\sum_{j=1}^{N} k_{j}=2 M$. Let

$$
G^{\mathbf{k}}:=\prod_{j=1}^{N}\left(h\left(q_{j}-q_{1}\right)-h * \rho_{t}\left(q_{1}\right)\right)^{k_{j}} .
$$

Then:

$$
\mathbb{E}_{t}\left[\left(\sum_{j=1}^{N}\left(h\left(q_{1}-q_{j}\right)-h * \rho_{t}\left(q_{1}\right)\right)\right)^{2 M}\right]=\sum_{\mathbf{k} \in \mathcal{M}}\left(\begin{array}{c}
2 M \\
\mathbf{k}
\end{array}\right) \mathbb{E}_{t}\left(G^{\mathbf{k}}\right) .
$$

Now we note that $\mathbb{E}_{t}\left(G^{\mathbf{k}}\right)=0$ whenever there exists a $1 \leq j \leq N$ such that $k_{j}=1$. This can be seen by integrating the $\mathrm{j}^{\prime}$ th variable first.

For the remaining terms, we have for any $1 \leq m \leq M$ :

$$
\int\left|h\left(q_{1}-q_{j}\right)\right|^{m} f_{t}\left(q_{j}, p_{j}\right) \mathrm{d}^{3} p_{j} \mathrm{~d}^{3} p_{j}=\int|h|^{m}\left(q_{1}-q_{j}\right) \rho_{t}\left(q_{j}\right) \mathrm{d}^{3} q_{j} .
$$

Now for $2 \leq \alpha<3$ and $m=1$ we estimate

$$
\begin{aligned}
& \left|h * \rho_{t}\left(q_{1}\right)\right| \leq \int|h|\left(q_{1}-y\right) \rho_{t}(y) \mathrm{d}^{3} y \\
& \leq c \int_{|y|<1}|y|^{-\alpha} \rho_{t}\left(q_{1}-y\right) \mathrm{d}^{3} y+c \int_{|y| \geq 1}\left|q_{j}\right|^{-\alpha} \rho_{t}\left(q_{1}-y\right) \mathrm{d}^{3} y \\
& \leq c\left(4 \pi\left\|\rho_{t}\right\|_{\infty}+\left\|\rho_{t}\right\|_{1}\right),
\end{aligned}
$$

while for $\alpha=3$, we find:

$$
\begin{aligned}
& \left|h * \rho_{t}\left(q_{1}\right)\right| \leq \int|h|\left(q_{1}-y\right) \rho_{t}(y) \mathrm{d}^{3} y \\
& \leq c\left(\int_{|y| \leq N^{-\delta}}+\int_{N^{-\delta}<|y|<1}+\int_{|y| \geq 1}\right)|h(y)| \rho_{t}\left(q_{1}-y\right) \mathrm{d}^{3} y \\
& \leq c\left\|\rho_{t}\right\|_{\infty} \int_{|y| \leq N^{-\delta}} N^{3 \delta} \mathrm{d}^{3} y+c\left\|\rho_{t}\right\|_{\infty} \int_{N^{-\delta}<|y|<1} \frac{1}{|y|^{3}} \mathrm{~d}^{3} y+c \int_{|y| \geq 1} \rho_{t}\left(q_{1}-y\right) \mathrm{d}^{3} y \\
& \leq c\left(4 \pi\left\|\rho_{t}\right\|_{\infty}\left(\frac{1}{3}+\log \left(N^{\delta}\right)\right)+\left\|\rho_{t}\right\|_{1}\right) .
\end{aligned}
$$

For $m \geq 2$, we find in any case

$$
\begin{aligned}
& \int|h|^{m}\left(q_{1}-y\right) \rho_{t}(y) \mathrm{d}^{3} y=\int|h|^{m}(y) \rho_{t}\left(q_{1}-y\right) \mathrm{d}^{3} y \\
\leq & \int_{|y|<N^{-\delta}}|h|^{m}(y) \rho_{t}\left(q_{1}-y\right) \mathrm{d}^{3} y+\int_{|y| \geq N^{-\delta}}|h|^{m}(y) \rho_{t}\left(q_{1}-y\right) \mathrm{d}^{3} y \\
\leq & c \rho_{t}\left\|_{\infty}\left(4 \pi N^{-3 \delta} N^{\alpha \delta m}+\int_{|y| \geq N^{-\delta}} \frac{1}{|y|^{\alpha m}} \mathrm{~d}^{3} y\right) \leq 8 \pi c\right\| \rho_{t} \|_{\infty} N^{(\alpha m-3) \delta} .
\end{aligned}
$$


Hence, setting $C_{\alpha}:=16 \pi c\left\|\rho_{t}\right\|_{\infty}\left(1+\mathbb{1}_{\{\alpha=3\}} \log (N)\right)$ we can conclude that for all $m \geq 2$ :

$$
\left|h\left(q_{j}-q_{i}\right)-h\left(q_{i}\right)\right|^{m} \leq C_{\alpha}^{m} N^{(\alpha m-3) \delta} .
$$

Now, for $\mathbf{k}=\left(k_{1}, k_{2}, \ldots, k_{N}\right) \in \mathcal{M}$, let $\# \mathbf{k}$ denote the number of $k_{j}$ with $\alpha k_{j} \neq 0$. Note that if $\# \mathbf{k}>M$, we must have $k_{j}=1$ for at least one $1 \leq j \leq N$, so that $\mathbb{E}_{t}\left(G^{\mathbf{k}}\right)=0$. For the other multiindices, we get (using that the particles are statistically independent):

$$
\begin{aligned}
\mathbb{E}_{t}\left(G^{\mathbf{k}}\right)=\mathbb{E}_{t} & {\left[\prod_{j=1}^{N}\left(k_{\delta}\left(q_{j}-q_{i}\right)-k * \rho_{t}\left(q_{i}\right)\right)^{k_{j}}\right] } \\
& \leq \prod_{j=1}^{N} \mathbb{E}_{t}\left[\left(\left|h\left(q_{j}-q_{i}\right)\right|+\left|h * \rho_{t}\left(q_{i}\right)\right|\right)^{k_{j}}\right] \\
& \leq \prod_{j=1}^{N} C_{\alpha}^{k_{j}} N^{\left(\alpha k_{j}-3\right) \delta} \\
& \leq C_{\alpha}^{2 M} N^{2 M \alpha \delta} N^{-3 \delta \# \mathbf{k}} .
\end{aligned}
$$

Finally, we observe that for any $l \geq 1$, the number of multiindices $\mathbf{k} \in \mathcal{M}$ with $\# \mathbf{k}=l$ is bounded by

$$
\sum_{\# \mathbf{k}=l} 1 \leq\left(\begin{array}{c}
N \\
l
\end{array}\right)(2 M)^{l} \leq(2 M)^{2 M} N^{l} .
$$

Thus:

$$
\begin{aligned}
\mathbb{P}_{t}\left(D_{1}\right) & \leq \frac{1}{N^{2 M(1-\beta)}} \sum_{\mathbf{k} \in \mathcal{M}}\left(\begin{array}{c}
2 M \\
\mathbf{k}
\end{array}\right) \mathbb{E}_{t}\left(G^{\mathbf{k}}\right) \\
& \leq C_{\alpha}^{2 M} C_{M} \frac{N^{2 M \alpha \delta}}{N^{2 M(1-\beta)}} \sum_{l=1}^{M} N^{(1-3 \delta) l} \\
& \leq C_{\alpha}^{2 M} M C_{M} N^{2 M(\alpha \delta+\beta-1)} \max \left\{N^{M(1-3 \delta)}, 1\right\} \\
& \leq C_{\alpha}^{2 M} M C_{M} N^{-\epsilon M}
\end{aligned}
$$

where $C_{M}$ is some constant depending on $M$ and

$$
\epsilon:= \begin{cases}1-2 \beta+\delta(3-2 \alpha) & \text { if } 3 \delta<1 \\ 2(1-\beta-\alpha \delta) & \text { if } 3 \delta \geq 1\end{cases}
$$

$\epsilon \geq 0$ according to (51). For $2 \leq \alpha<3$ we conclude the proof by noting that

$$
\mathbb{P}_{t}(D) \leq N \mathbb{P}_{t}\left(D_{1}\right) \leq C_{\alpha}^{2 M} M C_{M} N^{-(\epsilon M+1)},
$$

and choosing $M$ so large that $(\epsilon M-1)=\gamma$. For $\alpha=3$, however, equation (60) becomes

$$
\mathbb{P}_{t}(D) \leq C^{\prime}(M)(1+\log (N))^{2 M} N^{-(\epsilon M-1)},
$$


where $C^{\prime}(M)$ is some constant depending on $M$ and $\left\|\rho_{t}\right\|_{\infty}$. This can be rewritten as

$$
(1+\log (N))^{2 M} N^{-\epsilon M+1}=\left(\frac{1+\log (N)}{N^{\epsilon / 4}}\right)^{2 M} N^{-\frac{\epsilon}{2} M+1} .
$$

The function $g(x)=\frac{1+\log (x)}{x^{\epsilon / 4}}, x \in[1, \infty)$ is continuous with $\lim _{x \rightarrow \infty} g(x)=$ 0 . Hence, it has a maximum $C<+\infty$. In particular, $\frac{1+\log (N)}{N^{\epsilon / 4}} \leq C$ independent of $N$ and the announced result holds for $\alpha=3$, as well.

Corollary 7.3. Let $\mathcal{B}_{t}, \mathcal{C}_{t}$ as in Definition 7.1. Then we find for any $\gamma>0$ a constant $C_{\kappa}$ such that

$$
\begin{aligned}
& \mathbb{P}_{0}^{N}\left(\mathcal{B}_{t}\right) \geq 1-\frac{C_{\kappa}}{N^{\kappa}}, \\
& \mathbb{P}_{0}^{N}\left(\mathcal{C}_{t}\right) \geq 1-\frac{C_{\kappa}}{N^{\kappa}} .
\end{aligned}
$$

In other words, for any fixed $t$, initial conditions in $\mathcal{B}_{t} \cap \mathcal{C}_{t}$ are typical with the measure of "bad" initial conditions decreasing faster than any inverse power of $N$.

Proof. Note that

$$
\begin{aligned}
Z \in{ }^{N} \Phi_{t, 0}\left(\mathcal{B}_{t}\right) & \Longleftrightarrow\left|K_{\delta}^{N}(Z)-\bar{K}(Z)\right|_{\infty}<N^{-1+2 \delta} \\
& \Longleftrightarrow \max _{1 \leq i \leq N}\left|\frac{1}{N} \sum_{j \neq i}^{N} k_{\delta}^{N}\left(q_{i}-q_{j}\right)-k_{\delta}^{N} * \rho_{t}^{N}\left(q_{i}\right)\right|<N^{-1+2 \delta}
\end{aligned}
$$

and similarly

$$
\begin{aligned}
Z \in{ }^{N} \Phi_{t, 0}\left(\mathcal{C}_{t}\right) & \Longleftrightarrow\left|L_{\delta}^{N}(Z)-\bar{L}(Z)\right|_{\infty}<1 \\
& \Longleftrightarrow \max _{1 \leq i \leq N}\left|\frac{1}{N} \sum_{j \neq i}^{N} l_{\delta}^{N}\left(q_{i}-q_{j}\right)-l_{\delta}^{N} * \rho_{t}^{N}\left(q_{i}\right)\right|<1
\end{aligned}
$$

Applying the previous result once for $k_{\delta}^{N}$ with $\alpha=2$ and $\beta=1-2 \delta$ and once for $l_{\delta}^{N}$ with $\alpha=3$ and $\beta=0$, we get

$$
\begin{aligned}
& \mathbb{P}_{t}^{N}\left[\max _{1 \leq i \leq N}\left|\frac{1}{N} \sum_{j \neq i}^{N} k_{\delta}^{N}\left(q_{i}-q_{j}\right)-k_{\delta}^{N} * \rho_{t}^{N}\left(q_{i}\right)\right| \geq N^{-1+2 \delta}\right] \leq \frac{C_{\gamma}}{N^{\gamma}}, \\
& \mathbb{P}_{t}^{N}\left[\max _{1 \leq i \leq N}\left|\frac{1}{N} \sum_{j \neq i}^{N} l_{\delta}^{N}\left(q_{i}-q_{j}\right)-l_{\delta}^{N} * \rho_{t}^{N}\left(q_{i}\right)\right| \geq 1\right] \leq \frac{C_{\gamma}}{N^{\gamma}}
\end{aligned}
$$

Observing that $\mathbb{P}_{0}^{N}\left(\mathcal{B}_{t}\right)=\mathbb{P}_{t}^{N}\left(\Phi_{t, 0}\left(\mathcal{B}_{t}\right)\right)$ and $\mathbb{P}_{0}^{N}\left(\mathcal{C}_{t}\right)=\mathbb{P}_{t}^{N}\left(\Phi_{t, 0}\left(\mathcal{C}_{t}\right)\right)$, the statement follows. 


\section{Proof of Theorem 4.2}

We now have everything in place to prove our first Theorem 4.2 That is, we will prove the following statement:

Under the assumptions of Theorem 4.2, we find for all $\delta<\frac{1}{3}$ and $T>0$ a constant $C_{0}>0$ such that for any $\beta>0$ there exists a constant $C>0$ such that for $N \geq e^{\left(\frac{C_{0} T+1}{1-3 \delta}\right)^{2}}$

$$
\mathbb{P}_{0}\left[\sup _{0 \leq s \leq T}\left\{\Delta_{Z, s}^{N}\right\} \geq N^{-\delta}\right] \leq T \frac{C}{N^{\beta}}
$$

In order to control the evolution of $\sup _{0 \leq s \leq t}\left\{\Delta_{Z, s}^{N}\right\}$, respectively $J_{t}^{N, \lambda}(Z)$ defined in (40), we will need the following Lemma.

Lemma 8.1. For a function $g: \mathbb{R} \rightarrow \mathbb{R}$, we denote by

$$
\partial_{t}^{+} g(t):=\lim _{\Delta^{N} t \searrow 0} \frac{g\left(t+\Delta^{N} t\right)-g(t)}{\Delta^{N} t}
$$

the right-derivative of $f$ with respect to $t$. Let $g \in C^{1}(\mathbb{R})$ and $h(t):=$ $\sup _{0 \leq s \leq t} g(s)$. Then $\partial_{t}^{+} h(t)$ exists and $\partial_{t}^{+} h(t) \leq \max \left\{0, g^{\prime}(t)\right\}$ for all $t$.

Proof. We have to distinguish 3 cases.

1) If $g^{\prime}(t) \leq 0$, there exists $\Delta^{N} t>0$ such that $g(s) \leq g(t), \forall s \in$ $\left[t, t+\Delta^{N} t\right)$. Thus for all $t^{\prime} \in\left[t, t+\Delta^{N} t\right)$ we have $h\left(t^{\prime}\right):=\sup _{0 \leq s \leq t^{\prime}} g(s)=$ $\sup _{0 \leq s \leq t} g(s)=h(t)$ and $\partial_{t}^{+} h(t)=0$.

2) If $g(t)<h(t)$, there exists $\Delta^{N} t>0$ such that $g(s) \leq h(t) \forall s \in$ $\left(t-\Delta^{N} t, t+\Delta^{N} t\right)$. This means that $h$ is constant on $\left(t-\Delta^{N} t, t+\Delta^{N} t\right)$ so that, in particular, $\partial_{t}^{+} h(t)=0$.

3) If $g(t)=h(t)$ and $g^{\prime}(t)>0$, there exists $\Delta^{N} t>0$ such that $g$ is monotonously increasing on $\left(t-\Delta^{N} t, t+\Delta^{N} t\right)$. Hence, we have $h\left(t^{\prime}\right)=$ $\sup _{0 \leq s \leq t^{\prime}} g(s)=g\left(t^{\prime}\right)$ for all $t^{\prime} \in\left[t+\Delta^{N} t\right)$ and thus $\partial_{t}^{+} h(t)=g^{\prime}(t)$.

Proof of Theorem 4.2. Recall from Definition 5.1

$$
\begin{array}{r}
\Delta_{Z, t}^{N}=\left.\sqrt{\log (N)}\right|^{N} \Psi_{t, 0}^{1}(Z)-\left.{ }^{N} \Phi_{t, 0}^{1}(Z)\right|_{\infty} \\
+\left|{ }^{N} \Psi_{t, 0}^{2}(Z)-{ }^{N} \Phi_{t, 0}^{2}(Z)\right|_{\infty} .
\end{array}
$$

As announced, we introduce for any $\lambda>0$ and $N \in \mathbb{N}$ the process

$$
J_{t}^{N, \lambda}(Z):=\min \left\{1, \sup _{0 \leq s \leq t}\left\{e^{\lambda \sqrt{\log (N)}(T-s)}\left(N^{\delta} \Delta_{Z, s}^{N}+N^{3 \delta-1}\right)\right\}\right\} .
$$

We consider the expectation $\mathbb{E}_{0}\left(J_{t}^{N, \lambda}\right)$ which we split as follows:

$$
\mathbb{E}_{0}\left(J_{t}^{N, \lambda}\right)=\mathbb{E}_{0}\left(J_{t}^{N, \lambda} \mid \mathcal{A}_{t}^{c}\right)+\mathbb{E}_{0}\left(J_{t}^{N, \lambda} \mid \mathcal{A}_{t} \backslash \mathcal{B}_{t} \cap \mathcal{C}_{t}\right)+\mathbb{E}_{0}\left(J_{t}^{N, \lambda} \mid \mathcal{A}_{t} \cap \mathcal{B}_{t} \cap \mathcal{C}_{t}\right) .
$$


Here, $\mathcal{A}_{t}, \mathcal{B}_{t}, \mathcal{C}_{t}$ are the sets defined in Definition 7.1 and $J_{t}^{N, \lambda} \mid \mathcal{A}_{t}$ denotes the restriction of $J_{t}^{N, \lambda}$ to the set $\mathcal{A}_{t} \subset \mathbb{R}^{6 N}$ etc.

1) On $\mathcal{A}_{t}^{c}$, we have $\frac{\mathrm{d}}{\mathrm{d} t} J_{t}^{N, \lambda}(Z)=0$, since $J_{t}^{N, \lambda}(Z)$ is already maximal and thus also

$$
\frac{\mathrm{d}}{\mathrm{d} t} \mathbb{E}_{t}\left(J_{t}^{N, \lambda} \mid \mathcal{A}_{t}^{c}\right)=0 .
$$

2) For $Z \in \mathcal{A}_{t}$, we have to consider $\partial_{t}^{+} J_{t}^{N, \lambda}(Z) \leq \max \left\{0, I_{t}^{N, \lambda}(Z)\right\}$ with

$$
\begin{aligned}
I_{t}^{N, \lambda}(Z):= & \frac{d}{d t}\left(e^{\lambda \sqrt{\log (N)}(T-t)}\left(N^{\delta} \Delta_{Z, t}^{N}+N^{3 \delta-1}\right)\right) \\
= & -\lambda \sqrt{\log (N)} e^{\lambda \sqrt{\log (N)}(T-t)}\left(N^{\delta} \Delta_{Z, t}^{N}+N^{3 \delta-1}\right) \\
& +e^{\lambda \sqrt{\log (N)}(T-t)} N^{\delta} \partial_{t} \Delta_{Z, t}^{N} .
\end{aligned}
$$

We split $\partial_{t} \Delta_{Z, t}^{N}$ into

$$
\begin{aligned}
& \partial_{t}\left|{ }^{N} \Psi_{t, 0}^{1}(Z)-{ }^{N} \Phi_{t, 0}^{1}(Z)\right|_{\infty} \leq\left|\partial_{t}\left({ }^{N} \Psi_{t, 0}^{1}(Z)-{ }^{N} \Phi_{t, 0}^{1}(Z)\right)\right|_{\infty} \\
& \leq\left|{ }^{N} \Psi_{t, 0}^{2}(Z)-{ }^{N} \Phi_{t, 0}^{2}(Z)\right|_{\infty} \leq \sup _{0 \leq s \leq t}\left|{ }^{N} \Psi_{s, 0}^{2}(Z)-{ }^{N} \Phi_{s, 0}^{2}(Z)\right|_{\infty}
\end{aligned}
$$

and

$$
\begin{aligned}
\left.\partial_{t}\right|^{N} \Psi_{t, 0}^{2}(Z)-\left.{ }^{N} \Phi_{t, 0}^{2}(Z)\right|_{\infty} & \leq\left|\partial_{t}\left({ }^{N} \Psi_{t, 0}^{2}(Z)-{ }^{N} \Phi_{t, 0}^{2}(Z)\right)\right|_{\infty} \\
& \leq\left|K_{\delta}^{N}\left(\Psi_{t, 0}^{1}(Z)\right)-\bar{K}_{t}\left(\Phi_{t, 0}^{1}(Z)\right)\right|_{\infty} .
\end{aligned}
$$

We begin by controlling the contribution of "bad" initial conditions not contained in $\mathcal{B}_{t}$ and $\mathcal{C}_{t}$. Since $\left|k_{\delta}^{N}\right| \leq N^{2 \delta}$, the total force acting on each particle (with the $1 / N$-scaling) is also bounded as $\left|K_{\delta}^{N}(Z)\right|_{\infty} \leq$ $N^{2 \delta}$. The mean field force $\bar{K}$ is of order 1 , according to Lemma 3.4 and $\left.N^{\delta}\right|^{N} \Psi_{t, 0}^{2}(Z)-\left.{ }^{N} \Phi_{t, 0}^{2}(Z)\right|_{\infty} \leq 1$ since $Z \in \mathcal{A}_{t}$. In total, we thus have $\sup \left\{\left|\partial_{t}^{+} J_{t}^{N, \lambda}(Z)\right|: Z \in \mathcal{A}_{t}\right\} \leq C e^{\lambda \sqrt{\log (N)} T} N^{3 \delta}$ for some $C>0$. According to Prop. 7.2 the probability for $Z \notin \mathcal{B}_{t} \cap \mathcal{C}_{t}$ decreases faster than any power of $N$. Hence, we can find for any $\kappa>0$ a constant $C_{\kappa}$ (which may differ from the $C_{\kappa}$ in Proposition 7.2), such that

$$
\begin{aligned}
& \partial_{t}^{+} \mathbb{E}_{0}\left(J_{t}^{N, \lambda} \mid \mathcal{A}_{t} \backslash\left(\mathcal{B}_{t} \cap \mathcal{C}_{t}\right)\right) \\
& \leq \sup \left\{\left|\partial_{t}^{+} J_{t}^{N, \lambda}(Z)\right|: Z \in \mathcal{A}_{t}\right\} \mathbb{P}_{0}\left[\left(\mathcal{A}_{t} \cap \mathcal{B}_{t}\right)^{c}\right] \\
& \leq e^{\lambda \sqrt{\log (N) T}} \frac{C_{\kappa}}{N^{\kappa}}
\end{aligned}
$$

3) It remains to control the change of $J_{t}^{N, \lambda}$ for the typical initial conditions $Z \in \mathcal{A}_{t} \cap \mathcal{B}_{t} \cap \mathcal{C}_{t}$. To this end, we consider:

$$
\begin{aligned}
& \left|K_{\delta}^{N}\left(\Psi_{t, 0}^{1}(Z)\right)-\bar{K}_{t}\left(\Phi_{t, 0}^{1}(Z)\right)\right|_{\infty} \\
& \quad \leq\left|K_{\delta}^{N}\left(\Psi_{t, 0}^{1}(Z)\right)-K_{\delta}^{N}\left(\Phi_{t, 0}^{1}(Z)\right)\right|_{\infty} \\
& \quad+\left|K_{\delta}^{N}\left(\Phi_{t, 0}^{1}(Z)\right)-\bar{K}_{t}\left(\Phi_{t, 0}^{1}(Z)\right)\right|_{\infty} .
\end{aligned}
$$


Since $Z \in \mathcal{B}_{t}$, it follows that

$$
\left|K_{\delta}^{N}\left(\Phi_{t, 0}^{1}(Z)\right)-\bar{K}_{t}\left(\Phi_{t, 0}^{1}(Z)\right)\right|_{\infty}<N^{2 \delta-1} .
$$

For (72), we use the triangle to get for any $1 \leq i \leq N$ :

$$
\begin{aligned}
\left|\left(K_{\delta}^{N}\left(\Psi_{t, 0}^{1}(Z)\right)-K\left(\Phi_{t, 0}^{1}(Z)\right)\right)_{i}\right|_{\infty} & \leq\left|\sum_{j=1}^{N} k_{\delta}^{N}\left(\Psi_{i}^{1}-\Psi_{j}^{1}\right)-k_{\delta}^{N}\left(\Phi_{i}^{1}-\Phi_{j}^{1}\right)\right|_{\infty} \\
& \leq \sum_{j=1}^{N}\left|k_{\delta}^{N}\left(\Psi_{i}^{1}-\Psi_{j}^{1}\right)-k_{\delta}^{N}\left(\Phi_{i}^{1}-\Phi_{j}^{1}\right)\right|_{\infty} .
\end{aligned}
$$

Since $Z \in \mathcal{A}_{t}$, i.e. $J_{t}^{N, \lambda}(Z)<1$, we have in particular for $N>3$ i.e. $\log (N)>1, \sup _{0<s<t}\left|{ }^{N} \Psi_{s, 0}^{1}(Z)-{ }^{N} \Phi_{s, 0}^{1}(Z)\right|_{\infty}<N^{-\delta}$. Therefore we can use Lemma 6.3 to get the bound

$$
\begin{aligned}
\left|k_{\delta}^{N}\left(\Psi_{i}^{1}-\Psi_{j}^{1}\right)-k_{\delta}^{N}\left(\Phi_{i}^{1}-\Phi_{j}^{1}\right)\right|_{\infty} & \leq l_{\delta}^{N}\left(\Phi_{i}^{1}-\Phi_{j}^{1}\right)\left|\left(\Psi_{i}^{1}-\Psi_{j}^{1}\right)-\left(\Phi_{i}^{1}-\Phi_{j}^{1}\right)\right|_{\infty} \\
& \leq 2 l_{\delta}^{N}\left(\Phi_{i}^{1}-\Phi_{j}^{1}\right)\left|\Psi_{t, 0}^{1}-\Phi_{t, 0}^{1}\right|_{\infty} .
\end{aligned}
$$

Since $Z \in \mathcal{C}_{t}$, it follows with Lemma 6.1 that

$\sum_{j=1}^{N} l_{\delta}^{N}\left(\Phi_{i}^{1}-\Phi_{j}^{1}\right)=\left(L_{\delta}^{N}\left(\Phi_{t, 0}(Z)\right)_{i} \leq\left\|l_{\delta}^{N} * \rho_{t}^{N}(q)\right\|_{\infty}+1 \leq 2 C_{l} \log (N)\left(1+\left\|\rho_{t}^{N}\right\|_{\infty}\right)\right.$.

Hence, setting $C_{0}:=2 C_{l} C_{\rho}$, where $C_{\rho}$ is the uniform bound on the charge densities from (22), we have

$$
\frac{\mathrm{d}}{\mathrm{d} t}\left|\Psi_{t}^{2}(Z)-\Phi_{t, 0}^{2}(Z)\right|_{\infty} \leq C_{0} \log (N)\left|\Psi_{t, 0}^{1}(Z)-\Phi_{t, 0}^{1}(Z)\right|_{\infty}+N^{2 \delta-1}
$$

for $Z \in \mathcal{A}_{t} \cap \mathcal{B}_{t} \cap \mathcal{C}_{t}$. Together with (69), this yields:

$$
\begin{aligned}
\left.\partial_{t}^{+} \Delta_{Z, t}^{N}\right|_{\mathcal{A}_{t} \cap \mathcal{B}_{t} \cap \mathcal{C}_{t}} & \leq \sqrt{\log (N)} \frac{\mathrm{d}}{\mathrm{d} t}\left|\Psi_{t, 0}^{1}(Z)-\Phi_{t, 0}^{1}(Z)\right|_{\infty}+\frac{\mathrm{d}}{\mathrm{d} t}\left|\Psi_{t, 0}^{2}(Z)-\Phi_{t, 0}^{2}(Z)\right|_{\infty} \\
& \leq \sqrt{\log (N)}\left|\Psi_{t, 0}^{2}(Z)-\Phi_{t, 0}^{2}(Z)\right|_{\infty} \\
& +\left[C_{0} \log (N)\left|\Psi_{t, 0}^{1}(Z)-\Phi_{t, 0}^{1}(Z)\right|_{\infty}+N^{2 \delta-1}\right] \\
& \leq C_{0} \sqrt{\log N} \Delta_{Z, t}^{N}+N^{2 \delta-1}
\end{aligned}
$$

Plugging this into equation (68) and using that $C_{l}>4 \pi$ we have found $\partial_{t}^{+} J_{t}^{N, \lambda}(Z) \leq \max \left\{0, I_{t}^{N, \lambda}(Z)\right\}$ with

$$
\begin{aligned}
I_{t}^{N, \lambda}(Z) \leq & -\lambda \sqrt{\log (N)} e^{\lambda \sqrt{\log (N)}(T-t)}\left(N^{\delta} \Delta_{Z, t}^{N}+N^{3 \delta-1}\right) \\
& +e^{\lambda \sqrt{\log (N)}(T-t)} N^{\delta}\left(C_{0} \sqrt{\log N} \Delta_{Z, t}^{N}+N^{2 \delta-1}\right) \\
= & \sqrt{\log (N)} N^{\delta} e^{\lambda \sqrt{\log (N)}(T-t)} \\
& {\left[\left(C_{0}-\lambda\right) \Delta_{Z, t}^{N}+\left(\frac{1}{\sqrt{\log (N)}}-\lambda\right) N^{2 \delta-1}\right] . }
\end{aligned}
$$


Choosing $\lambda=C_{0}$ gives that this is negative. Hence, we have

$$
\partial_{t}^{+} \mathbb{E}_{0}\left(J_{t}^{N, \lambda} \mid \mathcal{A}_{t} \cap \mathcal{B}_{t} \cap \mathcal{C}_{t}\right)=0 .
$$

Together with (67) and (71) we have found:

$$
\partial_{t}^{+} \mathbb{E}_{0}\left(J_{t}^{N, \lambda}\right) \leq e^{\lambda \sqrt{\log (N)} T} \frac{C_{\kappa}}{N^{\kappa}}
$$

and thus

$$
\mathbb{E}_{0}\left(J_{t}^{N, \lambda}\right)-\mathbb{E}_{0}\left(J_{0}^{N, \lambda}\right)=\mathbb{E}_{0}\left(J_{t}^{N, \lambda}-J_{0}^{N, \lambda}\right) \leq T e^{\lambda \sqrt{\log (N)} T} \frac{C_{\kappa}}{N^{\kappa}},
$$

uniform in $t \in[0, T]$. Observing that $\Delta_{Z, 0}^{N}=0$, we have $J_{0}^{N, \lambda}(Z) \equiv$ $e^{\lambda \sqrt{\log (N)} T} N^{3 \delta-1}$. Now we shall demand

$$
N \geq N_{0}:=e^{\left(\frac{\lambda T+1}{1-3 \delta}\right)^{2}} \Rightarrow e^{\lambda \sqrt{\log (N)} T} N^{3 \delta-1} \leq \frac{1}{2} .
$$

(Here we exploited the fact that $e^{\sqrt{\log (N)}}$ grows slower than any power of $N$ ). The random variable $J_{t}^{N, \lambda}-J_{0}^{N, \lambda}=J_{t}^{N, \lambda}-e^{\lambda \sqrt{\log (N)} T} N^{3 \delta-1}$ is then certainly non-negative and it follows from (77) that:

$$
\mathbb{P}_{0}\left[J_{T}^{N, \lambda}(Z)-J_{0}^{N, \lambda}(Z) \geq \frac{1}{2}\right] \leq 2 T e^{\lambda \sqrt{\log (N)} T} \frac{C_{\kappa}}{N^{\gamma}} .
$$

However, if $J_{T}^{N, \lambda}(Z)-J_{0}^{N, \lambda}(Z)<\frac{1}{2}$, we have together with (78) that $J_{T}^{N, \lambda}(Z)<1$. And in this case, we can conclude from

$$
\begin{aligned}
& J_{T}^{N, \lambda}(Z)-J_{0}^{N, \lambda}(Z) \\
& =\sup _{0 \leq s \leq T}\left\{e^{\lambda \sqrt{\log (N)}(T-s)}\left(N^{\delta} \Delta_{Z, s}^{N}+N^{3 \delta-1}\right)\right\}-e^{\lambda \sqrt{\log (N)} T} N^{3 \delta-1} \\
& \geq N^{\delta} \sup _{0 \leq s \leq T} \Delta_{Z, s}^{N}-\frac{1}{2}
\end{aligned}
$$

the bound

$$
\begin{aligned}
\mathbb{P}_{0}\left[\sup _{0 \leq s \leq T}\left\{\Delta_{Z, s}^{N}\right\} \geq N^{-\delta}\right] & \leq \mathbb{P}_{0}\left[J_{T}^{N, \lambda}(Z)-J_{0}^{N, \lambda}(Z) \geq \frac{1}{2}\right] \\
& \leq 2 T e^{\lambda \sqrt{\log (N)} T} \frac{C_{\kappa}}{N^{\kappa}} \leq \frac{2 T C_{\kappa}}{N^{\kappa-1+3 \delta}} .
\end{aligned}
$$

For any given $\beta>0$, we can choose $\kappa:=\beta+1-3 \delta$ so that the result takes the form

$$
\mathbb{P}_{0}\left[\sup _{0 \leq s \leq T}\left\{\Delta_{Z, s}^{N}\right\} \geq N^{-\delta}\right] \leq \frac{T C}{N^{\beta}},
$$

with $C=2 C_{\kappa}$, as announced. 


\section{Controlling the mean field dynamics}

The previous theorem contains our main approximation result for the mean field dynamics. As explained in Section 5. two more steps remain in order to complete the proof of Theorem 4.4. First, we have to show that the solutions $f_{t}^{N}$ of the regularized Vlasov-Poisson equation (10) converge to a solution of the proper Vlasov-Poisson equation as the cutoff is lifted with $N \rightarrow \infty$. Second we have to prove the approximation of the continuous Vlasov-density by the discretized version $\mu_{0}^{N}\left[\Phi_{t, 0}(Z)\right]$. The proof of $f_{t}^{N} \rightarrow f_{t}$ follows the method of Loeper [13].

Proposition 9.1. Let $f_{0}$ satisfy the assumptions of Theorem 4.4. Let $f_{t}^{N}$ and $f_{t}$ be the solution of the regularized, respectively the unregularized Vlasov-Poisson equation with initial datum $f_{0}$. Then we have for $p \in[1, \infty]$ and $N>3$ :

$$
W_{p}\left(f_{t}^{N}, f_{t}\right) \leq N^{-\delta} e^{t 2 C_{0} \sqrt{\log N}},
$$

where $C_{0}$ depends on $\sup _{t, N}\left\{\left\|\rho_{t}^{N}\right\|_{\infty},\left\|\rho_{t}^{f}\right\|_{\infty}\right\}$.

Proof. Let $\rho_{t}^{N}:=\rho\left[f_{t}^{N}\right]$ and $\rho_{t}^{f}:=\rho\left[f_{t}\right]$ denote the charge density induced by $f_{t}^{N}$ and $f_{t}$, respectively. Let $\varphi_{t}^{N}=\left(Q_{t}^{N}, P_{t}^{N}\right)$ be the characteristic flow of $f_{t}^{N}$. For the (unregularized) Vlasov-Poisson equation, the corresponding vector-field is not Lipschitz. However, as we assume the existence of a solution $f_{t}$ with bounded density $\rho_{t}$, the mean field force $k * \rho_{t}$ does satisfy a Log-Lip bound of the form $\mid k * \rho_{t}(x)-k *$ $\rho_{t}(y)|\leq C| x-y \mid\left(1+\log ^{-}(|x-y|)\right)$, where $\log ^{-}(x)=\max \{0,-\log (x)\}$. This is sufficient to ensure the existence of a characteristic flow $\psi_{t, s}^{f}=$ $\left(Q_{t, s}^{f}, P_{t, s}^{f}\right)$ such that $f_{t}=\psi_{t, s}^{f} \# f_{s}$.

Since $W_{p} \leq W_{q}$ for $p \leq q$, it suffices to prove the statement for the infinite Wasserstein distance $W_{\infty}$. We consider $\pi_{0}(x, y):=f_{0}(x) \delta(x-$ $y) \in \Pi\left(f_{0}, f_{0}\right)$, which is the optimal coupling yielding $\left.W_{\infty}\left(f_{t}^{N}, f_{t}\right)\right|_{t=0}=$ $W_{\infty}\left(f_{0}, f_{0}\right)=0$. Let $\pi_{t}=\left(\varphi_{t, 0}^{N}, \psi_{t, 0}^{f}\right) \# \pi_{0}$. Then $\pi_{t} \in \Pi\left(f_{t}^{N}, f_{t}\right) \forall t \in$ $[0, T)$ and we can consider

$$
\begin{aligned}
D(t): & =\pi_{t}-\operatorname{esssup}\left\{\sqrt{\log (N)}\left|x^{1}-y^{1}\right|+\left|x^{2}-y^{2}\right|\right\} \\
& =\pi_{0}-\operatorname{esssup}\left\{\sqrt{\log (N)}\left|Q_{t, 0}^{N}(x)-Q_{t, 0}^{f}(y)\right|+\left|P_{t, 0}^{N}(x)-P_{t, 0}^{f}(y)\right|\right\} \\
& =f_{0}-\operatorname{esssup}\left\{\sqrt{\log (N)}\left|Q_{t, 0}^{N}(x)-Q_{t, 0}^{f}(x)\right|+\left|P_{t, 0}^{N}(x)-P_{t, 0}^{f}(x)\right|\right\},
\end{aligned}
$$

which is an upper bound on $W_{\infty}\left(f_{t}^{N}, f_{t}\right)$ for $N>3$. We compute:

$$
\begin{aligned}
\partial_{t}^{+} D(t) \leq & f_{0}-\operatorname{esssup}\left\{\sqrt{\log N}\left|P_{t}^{N}(x)-P_{t}^{f}(x)\right|\right. \\
& \left.+\left|k_{\delta}^{N} * \rho_{t}^{N}\left(Q_{t}^{N}(x)\right)-k * \rho_{t}^{f}\left(Q_{t}^{f}(x)\right)\right|\right\} \\
\leq & \sqrt{\log (N)} D(t) \\
& +f_{0}-\operatorname{esssup}\left|k_{\delta}^{N} * \rho_{t}^{N}\left(Q_{t}^{N}(x)\right)-k * \rho_{t}^{f}\left(Q_{t}^{f}(x)\right)\right| .
\end{aligned}
$$


Now note that

$$
\begin{aligned}
k * \rho_{t}^{f}\left(Q_{t}^{f}(x)\right) & =\int_{\mathbb{R}^{3}} k\left(Q_{t}^{f}(x)-q\right) \mathrm{d} \rho_{t}^{f}(q)=\int_{\mathbb{R}^{3} \times \mathbb{R}^{3}} k\left(Q_{t}^{f}(x)-q\right) \mathrm{d} f_{t}(q, p) \\
& =\int_{\mathbb{R}^{3} \times \mathbb{R}^{3}} k\left(Q_{t}^{f}(x)-Q_{t}^{f}(z)\right) \mathrm{d} f_{0}(z)
\end{aligned}
$$

and analogously for $k_{\delta}^{N} * \rho_{t}^{N}\left(Q_{t}^{N}(x)\right)$. Hence, we can write the interaction term as

$$
\begin{aligned}
& \left|k_{\delta}^{N} * \rho_{t}^{N}\left(Q_{t}^{N}(x)\right)-k * \rho_{t}^{f}\left(Q_{t}^{f}(x)\right)\right| \\
& =\left|\int_{\mathbb{R}^{3} \times \mathbb{R}^{3}}\left[k_{\delta}^{N}\left(Q_{t}^{N}(x)-Q_{t}^{N}(z)\right)-k\left(Q_{t}^{f}(x)-Q_{t}^{f}(z)\right)\right] \mathrm{d} f_{0}(z)\right| \\
& \leq \int_{\mathbb{R}^{3} \times \mathbb{R}^{3}}\left|k_{\delta}^{N}\left(Q_{t}^{N}(x)-Q_{t}^{N}(z)\right)-k_{\delta}^{N}\left(Q_{t}^{f}(x)-Q_{t}^{f}(z)\right)\right| \mathrm{d} f_{0}(z) \\
& +\int_{\mathbb{R}^{3} \times \mathbb{R}^{3}}\left|k_{\delta}^{N}\left(Q_{t}^{f}(x)-Q_{t}^{f}(z)\right)-k\left(Q_{t}^{f}(y)-Q_{t}^{f}(z)\right)\right| \mathrm{d} f_{0}(z)
\end{aligned}
$$

The second term (83) can be bounded as

$$
\begin{gathered}
\int\left|k_{\delta}^{N}\left(Q_{t}^{f}(y)-Q_{t}^{f}(z)\right)-k\left(Q_{t}^{f}(y)-Q_{t}^{f}(z)\right)\right| \mathrm{d} f_{0}(z) \\
\quad=\int\left|k_{\delta}^{N}\left(Q_{t}^{f}(y)-q\right)-k\left(Q_{t}^{f}(y)-q\right)\right| \rho_{t}^{f}(q) \mathrm{d} q \\
\leq\left\|k_{\delta}^{N}-k\right\|_{1}\left\|\rho_{t}^{f}\right\|_{\infty} \leq 4 \pi N^{-\delta}\left\|\rho_{t}^{f}\right\|_{\infty},
\end{gathered}
$$

where we used the fact that $k$ and $k_{\delta}^{N}$ differ only on a set of radius $N^{-\delta}$, so that $\left\|k_{\delta}^{N}-k\right\|_{1} \leq\left\|\left.k\right|_{|q| \leq N^{-\delta}}\right\|_{1}=4 \pi N^{-\delta}$. The term (82) can be bounded by first using the mean-value theorem as

$$
\begin{aligned}
& \left|k_{\delta}^{N}\left(Q_{t}^{N}(x)-Q_{t}^{N}(z)\right)-k_{\delta}^{N}\left(Q_{t}^{f}(y)-Q_{t}^{f}(z)\right)\right| \\
& \quad \leq\left(\left|\nabla k_{\delta}^{N}\left(Q_{t}^{N}(x)-Q_{t}^{N}(z)\right)\right|+\left|\nabla k_{\delta}^{N}\left(Q_{t}^{f}(x)-Q_{t}^{f}(z)\right)\right|\right) \\
& \quad \cdot\left(\left|Q_{t}^{N}(x)-Q_{t}^{f}(x)\right|+\left|Q_{t}^{N}(z)-Q_{t}^{f}(z)\right|\right) .
\end{aligned}
$$

Taking the integral with respect to $\mathrm{d} f_{0}(z)$, we estimate $\left|Q_{t}^{N}(z)-Q_{t}^{f}(z)\right|$ by the esssup and $\left\|\nabla k_{\delta}^{N}\right\|_{1}$ with Lemma 6.1. This yields:

$$
\text { (82) } \leq C_{0} \log (N)\left(\left|Q_{t}^{N}(x)-Q_{t}^{f}(x)\right|+f_{0}-\operatorname{esssup}\left|Q_{t}^{N}(z)-Q_{t}^{f}(z)\right|\right) \text {. }
$$

with $C_{0}:=2 C_{l} C_{\rho}$ as before and $C_{\rho}$ the uniform bound on the charge density from (22). Taking also the $f_{0}$-esssup over the $x$-variable, we get the bound

$$
\begin{aligned}
& 2 C_{0} \log (N) f_{0}-\operatorname{esssup}\left|Q_{t}^{N}(z)-Q_{t}^{f}(z)\right| \\
& \leq 2 C_{0} \sqrt{\log (N)} D(t) .
\end{aligned}
$$

Putting everything together, we have found

$$
\partial_{t}^{+} D(t) \leq 2 C_{0} \sqrt{\log (N)} D(t)+4 \pi\left\|\rho_{t}\right\|_{\infty} N^{-\delta} .
$$


Using Gronwall's lemma and the fact that $D(0)=0$, we get

$$
W_{\infty}\left(f_{t}^{N}, f_{t}\right) \leq D(t) \leq e^{t 2 C_{0} \sqrt{\log (N)}} N^{-\delta},
$$

from which the desired proposition follows.

A standard Gronwall argument yields the following result.

Proposition 9.2. Let $\varphi_{t}^{N}=(Q(t, \cdot), P(t, \cdot))$ the characteristic flow of $f_{t}^{N}$ defined by (12) and ${ }^{N} \Phi_{t, s}$ the lift to the $N$-particle phase-space defined in (14). Then we have for all $p \in[1, \infty)$ and $N>3$ :

$$
W_{p}\left(\mu_{0}^{N}\left[\Phi_{t, 0}(Z)\right], f_{t}^{N}\right) \leq \sqrt{\log N} W_{p}\left(\mu_{0}^{N}[Z], f_{0}\right) e^{t C_{0} \sqrt{\log N}} .
$$

Proof. For $Z \in \mathbb{R}^{6 N}$ let $\pi_{0}(x, y) \in \Pi\left(\mu_{0}^{N}, f_{0}\right)$ and define $\pi_{t}=\left(\varphi_{t}^{N}, \varphi_{t}^{N}\right) \# \pi_{0} \in$ $\Pi\left(\mu_{0}^{N}\left[\Phi_{t, 0}(Z)\right], f_{t}^{N}\right)$. Note that both measures are now transported with the same flow. Set

$$
\begin{aligned}
& D_{p}(t):=\left[\int_{\mathbb{R}^{6} \times \mathbb{R}^{6}}\left(\sqrt{\log N}\left|x^{1}-y^{1}\right|+\left|x^{2}-y^{2}\right|\right)^{p} \mathrm{~d} \pi_{t}(x, y)\right]^{1 / p} \\
= & {\left[\int_{\mathbb{R}^{6} \times \mathbb{R}^{6}}\left(\sqrt{\log N}\left|Q_{t}(x)-Q_{t}(y)\right|+\left|P_{t}(x)-P_{t}(y)\right|\right)^{p} \mathrm{~d} \pi_{0}(x, y)\right]^{1 / p} . }
\end{aligned}
$$

We compute:

$$
\begin{array}{r}
\frac{\mathrm{d}}{\mathrm{d} t} D_{p}^{p}(t)=p \int \mathrm{d} \pi_{0}(x, y)\left(\sqrt{\log N}\left|Q_{t}(x)-Q_{t}(y)\right|+\left|P_{t}(x)-P_{t}(y)\right|\right)^{p-1} \\
\left(\sqrt{\log N}\left|P_{t}(x)-P_{t}(y)\right|+\left|k_{\delta}^{N} * \rho_{t}^{N}\left(Q_{t}(x)\right)-k_{\delta}^{N} * \rho_{t}^{N}\left(Q_{t}(y)\right)\right|\right)
\end{array}
$$

Using again the Lipschitz bound

$$
\left|k_{\delta}^{N} * \rho_{t}^{N}\left(Q_{t}(x)\right)-k_{\delta}^{N} * \rho_{t}^{N}\left(Q_{t}(y)\right)\right| \leq C_{0} \log (N)\left|Q_{t}(x)-Q_{t}(y)\right|
$$

for $N>3$, i.e. $\log (N)>1$, we arrive at the estimate

$$
D(t) \leq D(0)+C_{0} \sqrt{\log N} \int D(s) \mathrm{d} s
$$

Hence by Gronwall's inequality:

$W_{p}\left(\mu_{0}^{N}\left[\Phi_{t, 0}(Z)\right], f_{t}^{N}\right)=W_{p}\left(\varphi_{t}^{N} \# \mu_{0}^{N}, \varphi_{t}^{N} \# f_{t}\right) \leq D(t) \leq D(0) e^{t C_{0} \sqrt{\log N}}$.

Taking on the right-hand side the infimum over all $\pi_{0}(x, y) \in \Pi\left(\mu_{0}^{N}, f_{0}\right)$,

$$
W_{p}\left(\mu_{0}^{N}\left[\Phi_{t, 0}(Z)\right], f_{t}^{N}\right) \leq \sqrt{\log N} W_{p}\left(\mu_{0}^{N}, f_{0}\right) e^{t C_{0} \sqrt{\log N}} .
$$

In view of (85), it remains to establish an upper bound on the typical rate of convergence for $W_{p}\left(\mu_{0}^{N}[Z], f_{0}\right) \rightarrow 0$. (Note that, other than that, the result of Proposition 9.2 is actually deterministic.) Fortunately, we can rely for this purpose on recent, partcularly strong concentration estimates obtained by Fournier and Guillin, 2014 [4]. 
Theorem 9.3 (Fournier and Guillin). Let $f$ be a probability measure on $\mathbb{R}^{n}$ such that $\exists k>2 p$ :

$$
M_{k}(f):=\int_{\mathbb{R}^{n}}|z|^{k} \mathrm{~d} f(z)<+\infty .
$$

Let $\left(Z_{i}\right)_{i=1, \ldots, N}$ be a sample of independent variables, distributed according to the law $f$ and consider $\mu_{0}^{N}[Z]:=\sum_{i=1}^{N} \delta_{Z_{i}}$. Then, for any $\epsilon>0$ there exist constants $c, C$ depending only on $k, M_{k}(f)$ and $\epsilon$ such that for all $N \geq 1$ and $\xi>0$ :

$$
\mathbb{P}_{0}\left[W_{p}^{p}\left(\mu^{N}, f\right)>\xi\right] \leq C N(N \xi)^{-\frac{k-\epsilon}{p}}+C \mathbb{1}_{\xi \leq 1} a(N, \xi),
$$

with

$$
a(N, \xi):= \begin{cases}\exp \left(-c N \xi^{2}\right) & \text { if } p>n / 2 \\ \exp \left(-c N\left(\frac{\xi}{\ln (2+1 / \xi)}\right)^{2}\right) & \text { if } p=n / 2 \\ \exp \left(-c N \xi^{n / p}\right) & \text { if } p \in[1, n / 2) .\end{cases}
$$

With these large deviation estimates, we get the following.

Corollary 9.4. Let $p \in[1, \infty), \gamma<\min \left\{\delta, \frac{1}{6}, \frac{1}{2 p}\right\}$ and $N>3$. Then there exists constants $c, C>0$ such that

$$
\begin{aligned}
\mathbb{P}_{0}\left[\exists t \in[0, T]: W_{p}\left(\mu_{0}^{N}\left[\Phi_{t, 0}(Z)\right], f_{t}\right)\right. & >\left(1+\sqrt{\log (N))} N^{-\gamma} e^{t 2 C_{0} \sqrt{\log N}}\right] \\
& \leq C\left(e^{-c N^{1-(6 \vee 2 p) \gamma}}+N^{1-\frac{k}{2 p}}\right)
\end{aligned}
$$

where we use the notation $6 \vee 2 p:=\max \{6,2 p\}$.

Proof. By assumption in Theorem 4.4, there exists $k>2 p$ such that $M_{k}\left(f_{0}\right)<+\infty$. Applying Theorem 9.3 with $\xi=N^{-p \gamma}, \epsilon=\frac{k(1-2 p \gamma)}{2(1-p \gamma)}$ and the finite-moment condition (1), we get constants $C, c>0$ such that

$$
\mathbb{P}_{0}\left[W_{p}\left(\mu_{0}^{N}[Z], f_{0}\right)>N^{-\gamma}\right] \leq C\left(e^{-c N^{1-(6 \vee 2 p) \gamma}}+N^{1-\frac{k}{2 p}}\right) .
$$

Thus, with Proposition 9.2, we conclude

$$
\begin{aligned}
\mathbb{P}_{0}\left[\exists t \in[0, T]: W_{p}\left(\mu_{0}^{N}\left[\Phi_{t, 0}(Z)\right], f_{t}^{N}\right)\right. & >\sqrt{\log (N)} N^{-\gamma} e^{\left.t C_{0} \sqrt{\log N}\right]} \\
\leq & C\left(e^{-c N^{1-(6 \vee 2 p) \gamma}}+N^{1-\frac{k}{2 p}}\right) .
\end{aligned}
$$

Adding the bound $W_{p}\left(f_{t}^{N}, f_{t}\right) \leq N^{-\delta} e^{t 2 C_{0} \sqrt{\log N}}$ from Proposition 9.1. the statement follows.

Now we have everything in place to complete the proof of Theorem 4.4 . 
Proof of Theorem 4.4. Let $p \in[1, \infty), \gamma<\frac{1}{6}$ and $N>3$. We split the approximation into

$$
\begin{aligned}
W_{p}\left(\mu_{t}^{N}[Z], f_{t}\right) & \leq W_{p}\left(\mu_{0}^{N}\left[\Psi_{t, 0}(Z)\right], \mu_{0}^{N}\left[\Phi_{t, 0}(Z)\right]\right) \\
& +W_{p}\left(\mu_{0}^{N}\left[\Phi_{t, 0}(Z)\right], f_{t}\right) .
\end{aligned}
$$

From Corollary 9.4 we get constants $c, C_{1}>0$ such that

$$
\begin{aligned}
\mathbb{P}_{0}\left[\exists t \in[0, T]: W_{p}\left(\mu_{0}^{N}\left[\Phi_{t, 0}(Z)\right], f_{t}\right)\right. & \left.>(1+\sqrt{\log N}) N^{-\gamma} e^{t 2 C_{0} \sqrt{\log (N)}}\right] \\
& \leq C_{1}\left(e^{-c N^{1-(6 \vee 2 p) \gamma}}+N^{1-\frac{k}{2 p}}\right) .
\end{aligned}
$$

In Theorem 4.2 we choose $\beta=\frac{k}{2 p}-1$ and get a constant $C^{\prime}$ so that, together with Lemma 5.2

$$
\mathbb{P}_{0}\left[\exists t \in[0, T]: W_{p}\left(\mu_{0}^{N}\left[\Psi_{t, 0}(Z)\right], \mu_{0}^{N}\left[\Phi_{t, 0}(Z)\right]\right) \geq N^{-\delta}\right] \leq T C^{\prime} N^{1-\frac{k}{2 p}}
$$

for any $N \geq e^{\left(\frac{C_{0} T+1}{1-3 \delta}\right)^{2}}$. Putting both estimates together and choosing $\gamma<\min \left\{\frac{1}{6}, \delta\right\}$, we have found that

$$
\begin{aligned}
\mathbb{P}_{0} & {\left[\exists t \in[0, T]: W_{p}\left(\mu_{t}^{N}[Z], f_{t}\right)>\right.} \\
& \left.N^{-\delta}+(1+\sqrt{\log N}) N^{-\gamma} e^{t 2 C_{0} \sqrt{\log (N)}}\right] \\
\leq & C_{1} e^{-c N^{1-(6 \vee 2 p) \gamma}}+C_{2} T N^{1-\frac{k}{2 p}}
\end{aligned}
$$

with $C_{2}:=C_{1}+C^{\prime}$. We can simplify this result by noting that $e^{\lambda \sqrt{\log (N)}} \leq N^{1-3 \delta}$ for $N \geq e^{\left(\frac{\lambda}{1-3 \delta}\right)^{2}}$. We shall thus demand $N \geq$ $N_{1}:=e^{\left(\frac{2\left(C_{0} T+1\right)}{1-3 \delta}\right)^{2}}$ which yields $N^{1-3 \delta} \geq \max \left\{e, 2(1+\sqrt{\log N}) e^{2 C_{0} T} \sqrt{\log (N)}\right\}$ and conclude that

$$
\begin{array}{r}
\mathbb{P}_{0}\left[\exists t \in[0, T]: W_{p}\left(\mu_{t}^{N}[Z], f_{t}\right)>N^{-\gamma+1-3 \delta}\right] \\
\leq C_{1} e^{-c N^{1-(6 \vee 2 p) \gamma}}+C_{2} T N^{1-\frac{k}{2 p}}
\end{array}
$$

\section{Weaker singularities, open questions}

While the present paper focuses on the Vlasov-Poisson equation, the method presented here can also be applied to interactions with milder singularities, see 11. For better comparison with other approaches, in particular the reference paper of Hauray and Jabin, 2013, [6], we shall state here the corresponding results without further proof. Generalization to higher dimensions would be straight-forward, as well.

We use the characterization of force kernels introduced in Def. 2.1.

Theorem 10.1. Let $\alpha<2$. Let $k$ satisfy a $S^{\alpha}$ condition and $k_{\delta}^{N}$ satisfy a $S_{\delta}^{\alpha}$ condition with the additional assumption (9) and

$$
\delta<\frac{1}{1+\alpha}
$$


Assume (for simplicity) that $f_{0} \in L^{1} \cap L^{\infty}\left(\mathbb{R}^{3} \times \mathbb{R}^{3}, \mathbb{R}^{+}\right)$, normalized to $\int f_{0}=1$, has compact support and let $f$ be the unique solution of the Vlasov equation with force kernel $k$. For $Z \in \mathbb{R}^{6 N}$, let $\mu_{t}^{N}[Z]$ the unique weak solution of the (regularized) Vlasov equation with force $k_{\delta}^{N}$ and initial data $\mu_{0}^{N}[Z]$. Then we have molecular chaos in the following sense: For any $\beta>0, \gamma \leq \min \left\{\frac{1}{6}, \delta\right\}$ and $T>0$ there exists constant $C_{1}, C_{2}$ such that

$$
\mathbb{P}_{0}\left[\exists t \in[0, T]: W_{1}\left(\mu_{t}^{N}[Z], f_{t}\right)>N^{-\gamma}\right] \leq C_{1} e^{-c N^{1-6 \gamma}}+T C_{2} N^{-\beta} .
$$

This can be compared to the results in [6], where a statement similar to (93) is derived for the case $1 \leq \alpha<2$ with a cut-off of order

$$
\delta<\frac{1}{6} \min \left\{\frac{1}{\alpha-1}, \frac{5}{\alpha}\right\} .
$$

For $\alpha \in[1,2)$, the upper bound on $\delta$ given by (94) ranges between $\frac{5}{6}$ and $\frac{1}{6}$, while our upper bound from (92) ranges between $\frac{1}{2}$ and $\frac{1}{3}$. In particular, it is interesting to note that the cut-off required in [6] is smaller than ours for $\alpha<\frac{7}{5}$ but larger for $\frac{7}{5}<\alpha<2$. This suggests that the probabilistic estimates presented here fare better for strong singularities - in the sense of admitting a significantly smaller cut-off - while the method proposed in [6] provides better controls for mild singularities.

Most notably, Hauray and Jabin are able to treat the case $0<\alpha<1$ with no cut-off at all by providing an explicit control on the minimal particle distance (in $(p, q)$-space, strictly speaking, while integrating the forces over small time-intervals). As it stands, our method requires in any case a regularization of the microscopic dynamics. Since it proves very effective in this setting, it would be interesting to investigate if it can be extended - or possibly combined with the approach of [6] - to further reduce the cut-off or, ideally, dispense with it altogether for sufficiently mild singularities.

\section{Acknowledgements}

We thank Maxime Hauray, Michael Kiessling, Detlef Dürr, Martin Kolb, Ana Cañizares, Samir Salem and Young-Pil Choi for valuable comments on earlier versions of the manuscript. 


\section{References}

[1] N. Boers and P. Pickl. On mean field limits for dynamical systems. Journal of Statistical Physics, pages 1-16, 2015.

[2] W. Braun and K. Hepp. The Vlasov dynamics and its fluctuations in the $1 / \mathrm{N}$ limit of interacting classical particles. Communications in Mathematical Physics, 56(2):101-113, 1977.

[3] R. L. Dobrushin. Vlasov equations. Functional Analysis and Its Applications, 13(2):115-123, 1979.

[4] N. Fournier and A. Guillin. On the rate of convergence in wasserstein distance of the empirical measure. Probability Theory and Related Fields, 162:1-32, 2014.

[5] F.A. Grünbaum. Propagation of chaos for the Boltzmann equation. Archive for Rational Mechanics and Analysis, 42:323-345, 1971.

[6] M. Hauray and P.-E. Jabin. Particles approximations of Vlasov equations with singular forces: Propagation of chaos. To appear in Annales scientifiques de l'École Normale Supérieure, 2013.

[7] E. Horst. Global strong solutions of Vlasov's equation - necessary and sufficient conditions for their existence. Banach Center Publications, 19(1):143-153, 1987.

[8] E. Horst. On the asymptotic growth of the solutions of the VlasovPoisson system. Mathematical Methods in the Applied Sciences, 16:75-85, 1993.

[9] M. Kac. Foundations of kinetic theory. In Proceedings of the Third Berkeley Symposium on Mathematical Statistics and Probability, 1954-1955, volume vol III, pages 171-197. University of California Press, 1956.

[10] M. K.-H. Kiessling. The microscopic foundations of Vlasov theory for jellium-like Newtonian N-body systems. Journal of Statistical Physics, 155(6):1299-1328, 2014.

[11] D. Lazarovici. The Vlasov-Poisson dynamics as the mean-field limit of rigid charges. Preprint: arXiv:1502.07047, 2015.

[12] P.-L. Lions and B. Perthame. Propagation of moments and regularity for the 3-dimensional Vlasov-Poisson system. Inventiones Mathematicae, 105:415-430, 1991.

[13] G. Loeper. Uniqueness of the solution to the Vlasov-Poisson system with bounded density. Journal de Mathématiques Pures et Appliquées, 86:68-79, 2006.

[14] H. Neunzert. An introduction to the nonlinear Boltzmann-Vlasov equation. In C. Cercignani, editor, Kinetic Theories and the Boltzmann Equation, volume 1048 of Lecture Notes in Mathematics, pages 60-110. Springer, Berlin, Heidelberg, 1984.

[15] H. Neunzert and J. Wick. Die Approximation der Lösung von Integro-Differentialgleichungen durch endliche Punktmengen. 
In R. Ansorge and W. Törnig, editors, Numerische Behandlung nichtlinearer Integrodifferential - und Differentialgleichungen, volume 395 of Lecture Notes in Mathematics, pages 275-290. Springer, Berlin, Heidelberg, 1974.

[16] K. Pfaffelmoser. Global classical solutions of the Vlasov-Poisson system in three dimensions for general initial data. Journal of Differential Equations, 95(2):281-303, 1990.

[17] J. Schaeffer. Global existence of smooth solutions to the VlasovPoisson system in three dimensions. Communications in Partial Differential Equations, 16(8-9):1313-1335, 1991.

[18] H. Spohn. Dynamics of Charged Particles and their Radiation Field. Cambridge University Press, Cambridge, 2004.

[19] A.-S. Sznitman. Topics in propagation of chaos. In École d'Été de Probabilités de Saint-Flour XIX - 1989, volume 1464 of Lecture Notes in Mathematics, pages 165-251. Springer, Berlin, 1991.

[20] C. Villani. Optimal Transport Old and New, volume 338 of Grundlehren der mathematischen Wissenschaften. Springer, Berlin, 2009. 